\title{
Fluid-structure interaction simulations of cerebral arteries modeled by isotropic and anisotropic constitutive laws
}

\author{
Paolo Tricerri • Luca Dedè · Simone Deparis • \\ Alfio Quarteroni · Anne M. Robertson · Adélia Sequeira
}

Received: 20 September 2014 / Accepted: 22 December 2014 / Published online: 21 January 2015

(C) Springer-Verlag Berlin Heidelberg 2015

\begin{abstract}
This paper considers numerical simulations of fluid-structure interaction (FSI) problems in hemodynamics for idealized geometries of healthy cerebral arteries modeled by both nonlinear isotropic and anisotropic material constitutive laws. In particular, it focuses on an anisotropic model initially proposed for cerebral arteries to characterize the activation of collagen fibers at finite strains. In the current work, this constitutive model is implemented for the first time in the context of an FSI formulation. In this framework, we investigate the influence of the material model on the numerical results and, in the case of the anisotropic laws, the importance of the collagen fibers on the overall mechanical behavior of the tissue. With this aim, we compare our numerical results by analyzing fluid dynamic indicators, vessel wall displacement, Von Mises stress, and deformations of the collagen fibers. Specifically, for an anisotropic model with collagen fiber recruitment at finite strains, we highlight the progressive activation and deactivation processes of the fibrous compo-
\end{abstract}

P. Tricerri $(\bowtie) \cdot$ L. Dedè · S. Deparis · A. Quarteroni École Polytechnique Fédérale de Lausanne, Av. Piccard, Station 8, 1015 Lausanne, Switzerland e-mail: i.paolo.tricerri@gmail.com

P. Tricerri · A. Sequeira

Department of Mathematics and CEMAT, Instituto Superior

Técnico, Universidade de Lisboa, Av. Rovisco Pais 1,

1049-001 Lisbon, Portugal

A. Quarteroni

MOX - Modeling and Scientific Computing, Dipartimento di Matematica "F. Brioschi", Politecnico di Milano, via Bonardi 9, 20133 Milano, Italy

\section{A. M. Robertson}

Department of Mechanical Engineering and Materials Science, Department of Bioengineering, University of Pittsburgh, 3700 O'Hara Street, Pittsburgh, PA 15261, USA nent of the tissue throughout the wall thickness during the cardiac cycle. The inclusion of collagen recruitment is found to have a substantial impact on the intramural stress, which will in turn impact the biological response of the intramural cells. Hence, the methodology presented here will be particularly useful for studies of mechanobiological processes in the healthy and diseased vascular wall.

Keywords Material constitutive models - Cerebral arterial tissue $\cdot$ Hypereslatic isotropic laws $\cdot$ Hyperelastic anisotropic laws $\cdot$ Fluid-structure interaction $\cdot$ Numerical simulations

\section{Introduction}

The use of mathematical models and numerical simulations for the study of the blood flow in arteries and, in general, of the cardiovascular system at large, both in physiological and pathological conditions, has received an increasing attention in the Biomedical community during the last two decades [30]. Indeed, such study aims at enhancing the current knowledge of the physiology of the cardiovascular system, as well as at providing reliable tools to the medical doctors in order to predict the natural course of pathologies and, possibly, the occurrence of cardiovascular accidents $[15,30,48,74]$.

In this work, we focus on idealized geometries of blood vessels that represent healthy (physiological) conditions of the cerebral vasculature; indeed, we report that in the Biomedical community few works have focused so far on the study of the cerebral blood circulation in physiological conditions, as in e.g. $[24,49,66,90]$, while the majority of the contributions, namely $[8,29,31,40,53,56,62,81,84,90]$, dealt with developed cardiovascular diseases, as cerebral aneurysms; blood flow dynamics is simulated either in rigid arteries, as in $[29,31,53,66,90]$, or in compliant arteries 
[7, 8, 24,46, 81, 83, 84, 87]. In literature, different techniques, such as the Arbitrary Lagrangian-Eulerian (ALE) formulation [30], the immersed boundary method [18], or the ALEVMS method [76,77] have been proposed to formulate and solve the coupled fluid-structure interaction (FSI) problem. In addition, several significant aspects associated to the solution of the FSI problem have been addressed in recent years, as for example the computation of so-called estimated zero pressure state [78], the impact of the inflow boundary condition on the numerical results $[17,58]$, and the computation of meaningful hemodynamical indicators such as the Wall Shear Stress or the Oscillatory Shear Index [79]. The numerical results of FSI simulations have been analyzed in terms of the flow patterns inside the blood vessels $[24,49,66]$, or in developed aneurysms $[2,29,31,40,56]$, or by studying the distribution of mechanical stresses inside the aneurysm dome $[7,8,46,84]$. Furthermore, some contributions have focused on the influence on the numerical results of the geometrical configuration $[1,14,53,56,83,90]$ (e.g. addressing the impact of the image reconstruction process on the creation of the vascular computational domains on the blood flow patterns), or of the boundary conditions modeling the physiological blood flow at the inlet and outlet surfaces of the computational domains $[62,81]$. However, few works, namely $[19,82,84,86,87]$, have addressed the influence of the vessel wall constitutive model on the numerical results and associated hemodynamical indicators; more specifically, they highlight the relevance of the modeling choice for the arterial tissue in FSI simulations, for which non-negligible effects on the vessel wall deformations are reported when employing different constitutive laws. Nevertheless, we remark that, when FSI simulations are carried out, the arterial tissue is often still described as an isotropic material by means of both linear and nonlinear models; see e.g. [7,22,46,55,87].

In this work, we address the influence of the constitutive model for the arterial tissue on the FSI numerical results by considering both nonlinear isotropic and anisotropic constitutive models, the latter representing the state of the art of the cerebral arterial tissue modeling $[6,12,23,32,33,42,50$, $52,86,88]$. Since the arterial tissue is mainly composed of elastin, collagen fibers, and smooth muscle cells and features a layered structure [60], we consider constitutive models that describe the passive mechanical response of arteries which is related to the load bearing properties of elastin and collagen fibers [42]. We focus on constitutive models that can capture both the nonlinear behavior of arteries, especially at finite strains $[42,73]$, and, for some of the laws under consideration, their anisotropic mechanical response [60]. In our analysis, we consider isotropic constitutive models since they are still largely used in the Computational Mechanics community due to their capability of describing the nonlinear mechanical response of the tissue without requiring information on its fibrous nature. Anisotropic models are how- ever receiving increasing attention due to the advancements in microscopy techniques which provide insights regarding the orientation, distribution and mechanical contribution of the collagen fibers to the overall mechanical behavior of the tissue $[39,70]$.

For anisotropic models that describe the arteries as a fiber reinforced material, the collagen fibers are considered to be mechanically active, i.e. effectively mechanically responding to applied external loads, only when stretched beyond a minimum threshold, also called the activation stretch. In literature, two approaches have been proposed to describe such recruitment of the collagen fibers: the most common considers the collagen fibers as active as soon as the strain is greater than zero, as in $[32,42]$, and another one for which the activation of the collagenous component occurs only at a minimum stretched length of the fibers $[50,52,89]$. We remark that the recruitment of the collagen fibers at finite strains has been hypothesized as the underlying mechanism of the mechanical stiffening for increasing stresses in arteries $[11,39,42,67,73]$. Furthermore, the possibility of describing the level of activation of the collagen fibers according to the deformations of the tissue during the cardiac cycle may provide important insights on the role of the collagen fibers in the arterial tissue response to hemodynamic loads. In this regard, it should be recalled, that the extracellular matrix of the arterial wall is continually maintained by the intramural vascular cells through a process of synthesis and degradation of this matrix, referred to as turnover. Further, these same cells act as sensors to the local mechanical and biochemical environment and modify their production/degradation of extracellular matrix components such as collagen fibers, elastin fiber, proteoglycans and matrix metalloproteinases in response to these cues [36]. For example, fibroblasts in the vascular wall will change their gene expression and rate of collagen synthesis in response to the state of the matrix, which they can probe using adhesion contacts. Recently, in [35], it was demonstrated that the degree of anisotropy in a planar biaxial loading alters fibroblast orientation and proliferation, [35]. Furthermore, the vulnerability of collagen fibers to enzymatic degradation is known to be influenced by the level of mechanical strain to which they are exposed [9]. Such enzymatic degradation is common in the arterial wall for both normal collagen turnover as well as disease related processes. There is therefore a need to more accurately model the local collagen fiber orientation and loading state in studies in order to more accuately model the interplay between mechanics and biology in both heathy and diseased vessels [69]. The current work provides an important step in this direction, by developing and implementing a methodology for the computational simulation of collagen recruitment and deformations during the cardiac cycle in physiological conditions of blood flow in idealized arteries. 
The FSI numerical results obtained with the different constitutive laws are analyzed by comparing both the blood flow and vessel wall dynamics. In particular, the arterial tissue models are compared in terms of the vessel wall displacement and Von Mises stress, as done in e.g. [19,82], while the anisotropic models are also analyzed in terms of the collagen fibers deformations over the cardiac cycle; for the latter models, we highlight their potential within the context of FSI numerical simulations. We anticipate that our analysis indicates that the blood flow dynamics in the cerebral arteries is not significantly affected by the choice of the material model, provided that the latter is properly calibrated. Conversely, the material model chosen for the mechanical characterization of the tissue significantly affects the displacement magnitude and the mechanical stresses in the vessel wall during the cardiac cycle.

This paper is organized as follows. Section 2 introduces the coupled FSI problem that governs the blood flow in compliant arteries and the constitutive models under consideration. In addition, it details the set of boundary conditions used to simulate the physiological blood flow dynamics during the cardiac cycle. Section 3 deals with the numerical simulations carried out on two idealized geometries of blood vessels; it presents the material parameters for the different constitutive models, the computational domains, and briefly addresses the numerical discretization of the coupled FSI problem. Afterwards, the numerical results obtained in the case of a cylindrical and a toroidal geometry are presented and critically discussed. Finally, we draw some conclusions and discuss possible further developments of this work.

\section{Vascular fluid-structure interaction modeling}

This section introduces the system of partial differential equations modeling the blood flow in compliant arteries together with the constitutive models for the arterial wall under consideration and includes the FSI equations. The Navier-Stokes equations model the blood flow, while the conservation equation of linear momentum governs the deformations of the arterial wall under the action of the hemodynamical forces [30]. The fluid equations are formulated in the Eulerian (spatial) coordinates, while the ones for the vessel wall are formulated in the Lagrangian (material) coordinates; for this reason, the two subsystems are coupled by means of an Arbitrary Lagrangian-Eulerian (ALE) formulation $[30,61]$.

This section is organized as follows. Section 2.1 presents the coupled FSI problem. Afterwards, Sect. 2.2 details the isotropic and anisotropic constitutive models for the description of the mechanical behavior of the healthy cerebral arterial tissue. Finally, Sect. 2.3 presents the set of boundary conditions considered for the simulation of the FSI problem.

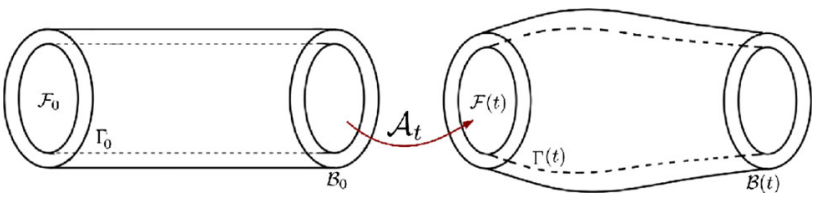

Fig. 1 Fluid and solid domains in the reference and current configurations of the FSI domain

\subsection{The coupled FSI problem}

When considering FSI problems in hemodynamics, the domain under consideration is composed of a fluid part (i.e. the vessel lumen) surrounded by a compliant solid part representing the arterial wall, both immersed in a threedimensional Euclidean space. In Fig. 1 the reference configurations, usually taken as the initial ones, of the fluid $(\mathcal{F})$ and solid $(\mathcal{B})$ domains, as well as of the fluid-structure interface $(\Gamma)$, are indicated by the subscript 0 , while the corresponding current configurations at a certain time $t>0$ are indicated as $\mathcal{F}(t), \mathcal{B}(t)$, and $\Gamma(t)$, respectively.

The geometric problem represents the displacement field of the vessel lumen, which is in material coordinates $\boldsymbol{X} \in \mathcal{F}_{0}$ and is computed from the one of the vessel wall at the fluidstructure interface $\Gamma$ [30]. As in [30], we indicate the displacement field in $\mathcal{F}_{0}$ as $\widehat{\boldsymbol{d}}_{\mathcal{F}}(\boldsymbol{X}, t): \mathcal{F}_{0} \times \mathbb{R}^{+} \rightarrow \mathbb{R}^{3}$ and we recall that a geometric condition, namely $\widehat{\boldsymbol{d}}_{\mathcal{F}}=\widehat{\boldsymbol{d}}_{\mid \Gamma_{0}}$ on $\Gamma_{0}$ at any time $t>0$, must be satisfied. Inside the fluid domain $\mathcal{F}_{0}$, the displacement field $\widehat{\boldsymbol{d}}_{\mathcal{F}}$ is defined as the harmonic extension of $\widehat{\boldsymbol{d}}_{\mid \Gamma_{0}}(t)$, as in $[21,22,30,55]$.

We assume the fluid to be Newtonian with constant viscosity and incompressible, as already done in e.g. $[7,8,16,68$, $86,87]$. The ALE formulation of the Navier-Stokes equations reads:

$\left\{\begin{array}{l}\nabla \cdot \boldsymbol{u}=0 \text { in } \mathcal{F}(t), \quad \forall t>0, \\ \left.\rho_{f} \frac{\partial \boldsymbol{u}}{\partial t}\right|_{\mathcal{A}_{t}}+\rho_{f}\left(\left(\boldsymbol{u}-\boldsymbol{w}_{\mathcal{F}}\right) \cdot \nabla\right) \boldsymbol{u} \\ -\nabla \cdot\left(\mu_{f}\left(\nabla \boldsymbol{u}+(\nabla \boldsymbol{u})^{T}\right)\right)+\nabla p=\mathbf{0} \text { in } \mathcal{F}(t), \quad \forall t>0,\end{array}\right.$

complemented with suitable initial and boundary conditions (see Sec. 2.3), where $(\boldsymbol{u}, p)$ represent the blood velocity and pressure, respectively, and $\boldsymbol{w}_{\mathcal{F}}$ indicate the ALE velocity of the fluid domain, defined as the time derivative of the vessel lumen displacement $\widehat{\boldsymbol{d}}_{\mathcal{F}}$; furthermore, $\rho_{f}$ and $\mu_{f}$ measure the density and dynamic viscosity of the blood, respectively. We remark that, in Eq. (1), $\nabla$ and $\nabla$. are the gradient and divergence operators in the Eulerian coordinates defined in the current configuration $\mathcal{F}(t)$.

The Lagrangian formulation of the conservation equation of linear momentum describing the deformations of the arterial wall reads: 
$\rho_{s} \frac{\partial \widehat{\boldsymbol{d}}}{\partial t}-\widehat{\nabla} \cdot(\boldsymbol{P}(\widehat{\boldsymbol{d}}))=\mathbf{0} \quad$ in $\mathcal{B}_{0}, \quad \forall t>0$,

with associated initial and boundary conditions (see Sect. 2.3), where $\rho_{s}$ indicates the arterial wall density, $\widehat{\boldsymbol{d}}$ represents the vessel wall displacement, and $\boldsymbol{P}(\widehat{\boldsymbol{d}})$ is the first PiolaKirchhoff tensor as a function of the arterial tissue displacement; $\widehat{\nabla}$. is the divergence operator in the material coordinates defined in the reference configuration $\mathcal{B}_{0}$ of the vessel wall. In this work, we model the arterial tissue as an hyperelastic material [41] whose mechanical behavior is fully characterized by means of the strain energy function per unit volume in the reference configuration $\mathcal{W}$, a scalar-valued function of the right Cauchy-Green tensor $\boldsymbol{C}$, defined in Sect. 2.2, i.e. $\mathcal{W}=\mathcal{W}(\boldsymbol{C})$. Consequently, the first Piola-Kirchhoff stress tensor in Eq. (2) depends on the material displacement field $\widehat{\boldsymbol{d}}$ through the deformation gradient $\boldsymbol{F}$ and right CauchyGreen tensors and on the strain energy function $\mathcal{W}$ according to the relation

$\boldsymbol{P}=2 \boldsymbol{F} \frac{\partial \mathcal{W}}{\partial \boldsymbol{C}}$.

We address the choice of the material constitutive models in Sect. 2.2.

To conclude, we recall that the fluid, solid and geometric problems are coupled by means of the three continuity conditions on the fluid-structure interface $\Gamma$. These are the geometric adherence condition, the continuity of the velocities:

$\boldsymbol{u}=\widehat{\boldsymbol{v}} \circ \mathcal{A}_{t}^{-1}$ on $\Gamma(t), \quad \forall t>0$,

where $\widehat{v}$ is the velocity of the arterial tissue, and the balance of the stresses

$$
\left(J_{\mathcal{A}_{t}} \sigma_{\mathcal{F}}(\boldsymbol{u}, p) \boldsymbol{F}_{\mathcal{A}_{t}}^{-T}\right) \widehat{\boldsymbol{n}}_{\mathcal{F}}+\boldsymbol{P}(\widehat{\boldsymbol{d}}) \widehat{\boldsymbol{n}}_{\mathcal{B}}=\mathbf{0} \quad \text { on } \Gamma_{0},
$$

for all $t>0$, where $\sigma_{\mathcal{F}}(\boldsymbol{u}, p)$ is the Cauchy stress tensor for a Newtonian fluid, $\boldsymbol{F}_{\mathcal{A}_{t}}^{-T}$ and $J_{\mathcal{A}_{t}}$ are the adjoint [41] and the determinant of the deformation gradient tensor defined from the ALE motion map, and $\widehat{\boldsymbol{n}}_{\mathcal{B}}$ and $\widehat{\boldsymbol{n}}_{\mathcal{F}}$ are the outward directed unit normal vectors to the solid domain $\mathcal{B}_{0}$ and the fluid $\mathcal{F}_{0}$ domain, respectively.

\subsection{Constitutive models for the arterial tissue}

In order to introduce different strain energy functions $\mathcal{W}$, we assume that there exists a continuous and smooth Lagrangian map $\phi_{t}$ which describes the movement of the arterial tissue from the reference configuration $\mathcal{B}_{0}$ into the current one $\mathcal{B}(t)$ for all times $t>0$. Consequently, the deformations and strains of the solid domain in the Lagrangian frame of reference are described by means of the deformation gradient tensor $\boldsymbol{F}:=\widehat{\nabla} \phi+\boldsymbol{I}$ and the Cauchy-Green tensor $\boldsymbol{C}:=\boldsymbol{F}^{T} \boldsymbol{F}$, respectively, with $\widehat{\nabla}$ denoting the gradient operator with respect to the Lagrangian coordinates $\widehat{\boldsymbol{X}}$ in $\mathcal{B}_{0}$ and $\boldsymbol{I}$ the identity second order tensor in $\mathbb{R}^{3}$. In the physiological range of deformations, the arterial tissue behaves as a nearly-incompressible material [13]; here, we adopt the approach based on the multiplicative volumetric-isochoric split of the deformation gradient $\boldsymbol{F}$ proposed in [28] and already employed in literature for cerebral arteries, as e.g. in $[8,46,85]$. The local deformation gradient $\boldsymbol{F}$ and the right Cauchy-Green tensor $\boldsymbol{C}$ are split into the so called volumetric and isochoric parts by means of the relations:

$\boldsymbol{F}=\left(J^{1 / 3} \boldsymbol{I}\right) \overline{\boldsymbol{F}}$ and $\boldsymbol{C}=\left(J^{2 / 3} \boldsymbol{I}\right) \overline{\boldsymbol{C}}$,

where $J:=\operatorname{det}(\boldsymbol{F})$ and the tensors $J^{1 / 3} \boldsymbol{I}$ and $J^{2 / 3} \boldsymbol{I}$ are associated with isotropic volume-changing deformations, while the tensors $\overline{\boldsymbol{F}}:=J^{-1 / 3} \boldsymbol{F}$ and $\overline{\boldsymbol{C}}:=J^{-2 / 3} \boldsymbol{C}$ with volume-preserving deformations of the material, for which $\operatorname{det}(\overline{\boldsymbol{F}}) \equiv \operatorname{det}(\overline{\boldsymbol{C}}) \equiv 1$. Based on Eq. (6), the strain energy function $\mathcal{W}$ is reformulated in its additive split form as:

$\mathcal{W}=\mathcal{W}(\boldsymbol{C}, J)=\mathcal{U}(J)+\overline{\mathcal{W}}(\overline{\boldsymbol{C}})$,

where the volumetric term $\mathcal{U}(J)$ penalizes the volumechanging deformations and the isochoric part $\overline{\mathcal{W}}(\overline{\boldsymbol{C}})$ characterizes the mechanical response of the material to external loads. From Eqs. (3) and (7), it follows that the first PiolaKirchhoff tensor $\boldsymbol{P}$ can be written as the sum of two terms, i.e. $\boldsymbol{P}=\widetilde{\boldsymbol{P}}+\overline{\boldsymbol{P}}$, where $\tilde{\boldsymbol{P}}$ and $\overline{\boldsymbol{P}}$ are referred to as the volumetric and isochoric first Piola-Kirchhoff stress tensors, respectively, and they are computed from the volumetric and isochoric strain energy functions, respectively, introduced in Eq. (7). We remark that we employ polyconvex strain energy functions of the state of deformation to ensure the existence of physically admissible solutions of Eq. (2) [41,72].

\subsubsection{Volumetric strain energy function}

The volumetric term $\mathcal{U}=\mathcal{U}(J)$ in Eq. (7) must be a strictly convex function of $J$ featuring with a unique global minimum at $J=1[37,41]$. In this work, it is assumed:

$\mathcal{U}(J)=\frac{\kappa}{4}\left[(J-1)^{2}+\log ^{2} J\right]$,

in order to penalize the cases $J \rightarrow \infty$ and $J \rightarrow 0$ corresponding to unphysical solutions [37]. The parameter $\kappa$, which can be interpreted as a bulk modulus, assumes the role of a user-specified penalty parameter that should be suitably tuned to weakly enforce the nearly incompressible response of the material in the physiological range of deformations of the body. 


\subsubsection{Isochoric isotropic and anisotropic strain energy functions}

The isochoric strain energy functions $\overline{\mathcal{W}}(\overline{\boldsymbol{C}})$ considered in this work are polyconvex functions for all the states of deformations. In order to describe the nonlinear behavior of cerebral arteries, and particularly the stiffening effect occurring at finite strains, we consider exponential type isotropic and anisotropic strain energy functions.

We consider two isotropic strain energy functions:

$\overline{\mathcal{W}}_{\mathrm{EXP} 1}(\overline{\boldsymbol{C}})=\frac{\alpha_{1}}{2 \gamma_{1}}\left(\mathrm{e}^{\gamma_{1}\left(I_{\overline{\boldsymbol{C}}}-3\right)}-1\right)$

firstly proposed in [23] and

$\overline{\mathcal{W}}_{\mathrm{EXP} 2}(\overline{\boldsymbol{C}})=\frac{\alpha_{2}}{2 \gamma_{2}}\left(\mathrm{e}^{\gamma_{2}\left(I_{\overline{\boldsymbol{C}}}-3\right)^{2}}-1\right)$,

considered in [3]. In Eqs. (9) and (10), $I_{\overline{\boldsymbol{C}}}=\operatorname{Tr}(\overline{\boldsymbol{C}})$ and $\alpha_{1}, \alpha_{2}, \gamma_{1}, \gamma_{2}$ are material parameters; specifically, $\alpha_{1}$ and $\alpha_{2}$ measure the mechanical low strain stiffness of the arterial tissue, while $\gamma_{1}$ and $\gamma_{2}$ are representative of the level of nonlinearity of the mechanical response of the vessel wall.

The anisotropic mechanical properties of the arterial wall arise from aligned components within the wall such as collagen fibers and smooth muscle cells. For this reason, anisotropic models for the arterial wall often explicitly include a fibrous contribution in addition to an isotropic component $[42,75]$. In such structurally motivated models, the mechanical response of the wall arises as the sum of contributions from the isotropic medium and fibrous components. The isotropic component is used to model contributions from elastin and other extracellular matrix components such as glycosaminoglycans (see, e.g. $[45,69])$. For simplicity, we simply refer to this isotropic component as the elastin contribution. Here, the fibrous contribution is modeled as the sum of the mechanical responses of a finite number of families of collagen fibers $[4,41,50,52,75]$. When modeling the arterial tissue as a fiber-reinforced material, the local spatial orientation of each family of fibers is assumed to be described by means of a material vectorial field $\widehat{\boldsymbol{a}}_{0}(\widehat{\boldsymbol{X}})$ of unit length in $\mathcal{B}_{0}$ $[41,75]$, as in Fig. 2. In this work we assume that two families of fibers are immersed in the background material (see Fig. 2), as e.g. in $[10,32,50]$, and that each of them forms an angle $\beta^{(i)}$ with the circumferential direction of the vessel wall, for $i=1,2$, that will change as the vessel wall is deformed. In this work, we consider the case where the collagen fibers may be undulated and will not commence load bearing until they are recruited. This condition is implemented via an activation criterion $[50,89]$. In particular, it will be assumed that these fibers will not be load bearing unless their stretch is larger than a certain threshold, called the activation stretch. Given

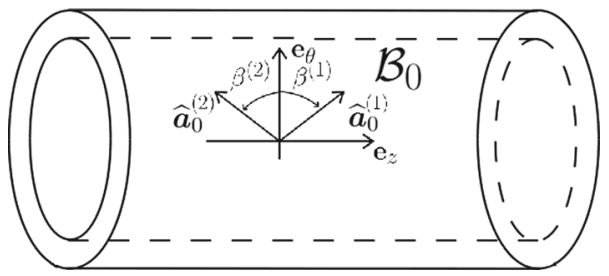

Fig. 2 Directions $\widehat{\boldsymbol{a}}_{0}, i=1,2$, of the families of collagen fibers in the reference configuration $\mathcal{B}_{0}$

the $i$-th material vectorial field $\widehat{\boldsymbol{a}}_{0}^{(i)}(\widehat{\boldsymbol{X}})$ in $\mathcal{B}_{0}$, the deformed vectorial field $\widehat{\boldsymbol{a}}_{t}^{(i)}$, representing the collagen fibers in the current configuration $\mathcal{B}(t)$, is defined as $\widehat{\boldsymbol{a}}_{t}^{(i)}=\boldsymbol{F} \widehat{\boldsymbol{a}}_{0}^{(i)}$ for all the families of fibers; here, when the activation stretch is reached, the corresponding configuration of the collagen fibers field will be indicated as $\boldsymbol{a}_{A}^{(i)}$, for $i=1,2$. We remark that the $i$-th family of collagen fibers is considered mechanically active when its stretch, defined by means the scalar quantity $\bar{I}_{4}^{(i)}=\overline{\boldsymbol{C}}:\left(\widehat{\boldsymbol{a}}_{0}^{(i)} \otimes \widehat{\boldsymbol{a}}_{0}^{(i)}\right)$ is larger than $\left\|\boldsymbol{a}_{A}^{(i)}\right\|^{2}$ that indicates the activation stretch of the single family of fibers which was aligned along $\widehat{\boldsymbol{a}}_{0}^{(i)}$ in $\mathcal{B}_{0}[3,50]$. When the collagen fibers are mechanically active, the isochoric anisotropic strain energy function describing the tissue reads:

$$
\begin{aligned}
\overline{\mathcal{W}} & \left(\overline{\boldsymbol{C}}, \widehat{\boldsymbol{a}}_{0}^{(1)}, \widehat{\boldsymbol{a}}_{0}^{(2)} ;\left\|\boldsymbol{a}_{A}^{(1)}\right\|^{2},\left\|\boldsymbol{a}_{A}^{(2)}\right\|^{2}\right) \\
& =\overline{\mathcal{W}}_{\text {elastin }}(\overline{\boldsymbol{C}})+\sum_{i=1}^{2} \overline{\mathcal{W}}_{\text {fiber }}^{(i)}\left(\overline{\boldsymbol{C}}, \widehat{\boldsymbol{a}}_{0}^{(i)} ;\left\|\boldsymbol{a}_{A}^{(i)}\right\|^{2}\right),
\end{aligned}
$$

where $\overline{\mathcal{W}}_{\text {elastin }}(\overline{\boldsymbol{C}})$ is the strain energy function describing the elastin which we set $\overline{\mathcal{W}}_{\text {elastin }}=\overline{\mathcal{W}}_{\text {EXP1 }}$, while $\overline{\mathcal{W}}_{\text {fiber }}^{(i)}\left(\overline{\boldsymbol{C}}, \widehat{\boldsymbol{a}}_{0}^{(i)} ;\left\|\boldsymbol{a}_{A}^{(i)}\right\|^{2}\right)$ is the strain energy function modeling the mechanical behavior of the single family of fibers for which the activation stretch of the single family of fibers is a characteristic parameter which should be obtained from experimental studies of the histology of the arterial tissue [39]. We consider $\overline{\mathcal{W}}_{\text {fiber }}^{(i)}\left(\overline{\boldsymbol{C}}, \widehat{\boldsymbol{a}}_{0}^{(i)} ;\left\|\boldsymbol{a}_{A}^{(i)}\right\|^{2}\right)$ of the form:

$$
\begin{aligned}
\overline{\mathcal{W}}_{\text {fiber }}^{(i)}\left(\overline{\boldsymbol{C}}, \widehat{\boldsymbol{a}}_{0}^{(i)} ;\left\|\boldsymbol{a}_{A}^{(i)}\right\|^{2}\right) & =\overline{\mathcal{W}}_{\text {fiber }}^{(i)}\left(\bar{I}_{4}^{(i)} ;\left\|\boldsymbol{a}_{A}^{(i)}\right\|^{2}\right) \\
& =\frac{\alpha^{(i)}}{2 \gamma^{(i)}}\left(e^{\gamma^{(i)}\left(\bar{I}_{4}^{(i)}-\left\|\boldsymbol{a}_{A}^{(i)}\right\|^{2}\right)^{2}}-1\right),
\end{aligned}
$$

where $\alpha^{(i)}$ and $\gamma^{(i)}$ represent the mechanical low strain stiffness and level of nonlinearity characterizing the $i$-th family of collagen fibers, respectively. In this work, we consider two different types of models for all the families of fibers. The first one corresponds to the case for which the activation stretch $\left\|\boldsymbol{a}_{A}^{(i)}\right\|^{2}$ is equal to one (immediate recruitment) for all the 
families of fibers [3,32]; correspondingly, we set in Eq. (12) $\left\|\boldsymbol{a}_{A}^{(i)}\right\|^{2}=1$ and the associated strain energy function, which we denote as $\overline{\mathcal{W}}_{\text {EXP2-ZS }}^{(i)}$, is:

$$
\begin{aligned}
\overline{\mathcal{W}}_{\text {EXP2-ZS }}^{(i)}\left(\overline{\boldsymbol{C}}, \widehat{\boldsymbol{a}}_{0}^{(i)}\right) & =\overline{\mathcal{W}}_{\mathrm{EXP2-ZS}}^{(i)}\left(\bar{I}_{4}^{(i)}\right) \\
& =\frac{\alpha^{(i)}}{2 \gamma^{(i)}}\left(e^{\gamma^{(i)}\left(\bar{I}_{4}^{(i)}-1\right)^{2}}-1\right),
\end{aligned}
$$

where "ZS" stands for the activation of the fibers with respect to the unloaded reference configuration, i.e. the configuration at zero strains. The second anisotropic model, proposed in $[50,89]$, assumes that the recruitment of the $i$-th family of collagen fibers occurs at finite strains whenever $\bar{I}_{4}^{(i)}>\left\|\boldsymbol{a}_{\mathrm{FS}}^{(i)}\right\|^{2}$, where $\left\|\boldsymbol{a}_{\mathrm{FS}}^{(i)}\right\|$ is the activation length associated to the $i$-th family in a deformed configuration of the body $\mathcal{B}_{\mathrm{FS}}^{(i)}$, called the activation configuration. Therefore, in this case, we set $\left\|\boldsymbol{a}_{A}^{(i)}\right\|^{2}=\left\|\boldsymbol{a}_{\mathrm{FS}}^{(i)}\right\|^{2}$ and the strain energy function $\left(\overline{\mathcal{W}}_{\mathrm{EXP} 2-\mathrm{FS}}^{(i)}\right)$ for the single family of fibers reads:

$$
\begin{aligned}
& \overline{\mathcal{W}}_{\mathrm{EXP} 2-\mathrm{FS}}^{(i)}\left(\overline{\boldsymbol{C}}, \widehat{\boldsymbol{a}}_{0}^{(i)} ;\left\|\boldsymbol{a}_{\mathrm{FS}}^{(i)}\right\|^{2}\right) \\
& =\overline{\mathcal{W}}_{\mathrm{EXP2-FS}}^{(i)}\left(\bar{I}_{4}^{(i)} ;\left\|\boldsymbol{a}_{\mathrm{FS}}^{(i)}\right\|^{2}\right) \\
& =\frac{\alpha^{(i)}}{2 \gamma^{(i)}}\left(e^{\gamma^{(i)}\left(\bar{I}_{4}^{(i)}-\left\|\boldsymbol{a}_{\mathrm{FS}}^{(i)}\right\|^{2}\right)^{2}}-1\right),
\end{aligned}
$$

where "FS" stands for finite strains.

As described in $[5,43,44]$, in the case of anisotropic laws, the collagen fibers recruitment is included in the definition of the first Piola-Kirchhoff tensor when the activation condition $\bar{I}_{4}^{(i)}>\left\|\boldsymbol{a}_{A}^{(i)}\right\|^{2}$ on the single family of fibers is satisfied. Here, we take into account the recruitment of the collagenous component of the tissue by means of a smooth activation function of the stretch of the fibers $H\left(\bar{I}_{4}^{(i)}-\left\|\boldsymbol{a}_{A}^{(i)}\right\|^{2} ; \epsilon\right)$ that multiplies the mechanical contribution of the each family of collagen fibers, indicated as $\overline{\boldsymbol{P}}_{\text {fiber }}^{(i)}$. Therefore, in the case of anisotropic models, the isochoric component of $\boldsymbol{P}$, i.e. $\overline{\boldsymbol{P}}=2 \boldsymbol{F} \frac{\partial \overline{\mathcal{W}}}{\partial \boldsymbol{C}}$, reads:

$\overline{\boldsymbol{P}}=\overline{\boldsymbol{P}}_{\text {elastin }}+\sum_{i=1}^{2} H\left(\bar{I}_{4}^{(i)}-\left\|\boldsymbol{a}_{A}^{(i)}\right\|^{2} ; \epsilon\right) \overline{\boldsymbol{P}}_{\text {fiber }}^{(i)}$,

where, $\overline{\boldsymbol{P}}_{\text {elastin }}$ and $\overline{\boldsymbol{P}}_{\text {fiber }}^{(i)}$ are the mechanical contribution of the elastin and single family of fibers, respectively, $H\left(\bar{I}_{4}^{(i)}-\left\|\boldsymbol{a}_{A}^{(i)}\right\|^{2} ; \epsilon\right)=\left(\frac{1}{\pi} \operatorname{atan}\left(\epsilon\left(\bar{I}_{4}^{(i)}-\left\|\boldsymbol{a}_{A}^{(i)}\right\|^{2}\right)\right)+\frac{1}{2}\right)$, and $\epsilon$ is a dimensionless user-specified parameter which we choose as $\epsilon=5.0 \cdot 10^{5}$ to sharply model the abrupt recruitment of the collagen fibers. We remark that, for each family of fibers, the function $H\left(\bar{I}_{4}^{(i)}-\left\|\boldsymbol{a}_{A}^{(i)}\right\|^{2} ; \epsilon\right)$ is introduced in Eq. (15) to approximate the corresponding Heaviside func- tion of $\bar{I}_{4}^{(i)}$, centered around $\left\|\boldsymbol{a}_{A}^{(i)}\right\|^{2}$. The recruitment of the collagen fibers may also be modeled using other approaches, for instance by means of a probability density function of the activation stretch as in [39].

\subsection{Boundary and initial conditions for the coupled FSI problem}

The equations defining the FSI problem must be complemented with a set of suitable boundary conditions defining physiological inflow and outflow conditions for the fluid domain as well as representing the tethering tissues around the vessel wall. At the inflow section of the fluid domain in Fig. 1, we apply Dirichlet boundary conditions:

$\boldsymbol{u}=\boldsymbol{u}_{\text {inlet }} \quad$ on $\left.\partial \mathcal{F}(t)\right|_{\text {inlet }}, \quad \forall t>0$,

where $\left.\partial \mathcal{F}(t)\right|_{\text {inlet }}$ is the inlet surface of the current configuration of $\mathcal{F}$ and $\boldsymbol{u}_{\text {inlet }}$ is a time varying parabolic (first order Womersley) profile centered with respect to $\left.\partial \mathcal{F}(t)\right|_{\text {inlet }}$ and directed along its inward normal unit vector. We remark that, as it will be detailed in Sect. 3, the time evolution of $\boldsymbol{u}_{\text {inlet }}$ is computed from a representative physiological flux profile for an internal carotid artery (ICA) [2,57]; particularly, as in [2], the time evolution of the flux profile is described by means of a Fourier expansion of the experimental measurements reported in [57] and then rescaled with respect to the cross section are of the inlet surface of the domains under consideration.

On the outlet surface we apply resistance boundary conditions [66] of the form:

$\sigma_{\mathcal{F}} \boldsymbol{n}_{\mathcal{F}}=R Q(\boldsymbol{u}) \boldsymbol{n}_{\mathcal{F}}+p_{V} \boldsymbol{n}_{\mathcal{F}} \quad$ on $\left.\partial \mathcal{F}(t)\right|_{\text {outlet }}, \quad \forall t>0$,

where $R$ is the resistance coefficient employed to attain physiological values of blood pressure in the deformed fluid domain, as done in e.g. [7, 8,66,80,82,83], $Q(\boldsymbol{u})$ is the flow through the outlet surface $\left.\partial \mathcal{F}(t)\right|_{\text {outlet }}$ of $\mathcal{F}(t)$, and $p_{V}$ is the reference pressure.

On the external surface of the solid domain, we consider an elastic Robin boundary condition in order to mimic the presence of the tethering tissues around the vessel, as done in e.g. [20,21]. As discussed in [21], the mathematical formulation of the Robin boundary condition is:

$\boldsymbol{P} \widehat{\boldsymbol{n}}_{\mathcal{B}}+K \widehat{\boldsymbol{d}}+h \widehat{\boldsymbol{n}}_{\mathcal{B}}=\mathbf{0} \quad$ on $\partial \mathcal{B}_{0, \text { ext }}, \quad \forall t>0$,

where $\partial \mathcal{B}_{0, \text { ext }}$ is the external surface of the solid domain in the reference configuration $\mathcal{B}_{0}, K$ is the elastic coefficient, representing the volumetric stiffness of the surrounding tissues, and $h=h(\widehat{\boldsymbol{X}}, t)$ is a scalar function describing an applied external load. Here, the elastic coefficient in the Robin condition is determined such that physiological radial deformations of the vessel wall are obtained [25], while we set $h=0$. 
We remark that elastic Robin boundary conditions have been employed in e.g. [20,21], while in [54,55,59] a viscoelastic Robin boundary condition is applied. As pointed out in $[20,21]$, physiological displacements of the arterial wall can be obtained by considering elastic Robin boundary conditions. For this reason, due to the general lack of experimental data to estimate the parameters in the viscoelastic Robin conditions of $[54,55,59]$ for the cerebral vasculature, pure elastic Robin boundary condition have been preferred to viscoelastic Robin conditions on the external surface. At the boundaries $\partial \mathcal{B}_{0, \text { inlet }}$ and $\partial \mathcal{B}_{0, \text { outlet }}$, which correspond to the faces of $\mathcal{B}_{0}$ in proximity of the inlet and outlet boundaries of the fluid domain, we impose homogeneous essential boundary conditions for the displacement. We remark that this choice may induce unrealistic deformations of the vessel in proximity of the inlet and outlet faces of the domain; however, as we will see in Sect. 3, this effect is rather local since realistic displacements and stresses will be instead obtained in a large central portion of the artery. Another advantage of this choice is related to the fact that more realistic boundary conditions, as those for which only the axial component of the displacement is set to zero, may lead to different deformations of the inlet and outlet boundaries of the fluid domain for the constitutive laws under consideration; however, this would require the modification and adaption of the inlet flow condition (16) to each constitutive material under consideration and at each time step.

Finally, we recall that for the geometric problem, we set homogeneous Dirichlet boundary conditions on both the inflow and outflow sections of $\mathcal{F}_{0}$, as for instance in [84].

Regarding the initial conditions for the FSI coupled problem, we set the blood flow velocity and pressure as well as the fluid and solid displacements equal to zero. Consequently, the numerical results are affected by an initial transient phase before the proper physiological conditions are matched. This aspect is detailed in Sect. 3 .

\section{Numerical simulations}

This section presents and discusses the FSI numerical simulations for idealized cerebral arteries. Firstly, it summarizes the values of the material parameters employed for the blood and arterial wall constitutive models introduced in Sect. 2. The material parameters that will be presented in Sect. 3.1 have been obtained from data fitting of experimental measurements of the mechanical behavior of healthy anterior cerebral arteries of which the computational domains reproduce the physiological dimensions. In Sect. 3.2 we illustrate the space and time discretizations for our FSI simulations and, finally, Sect. 3.3 presents the numerical results obtained using different constitutive models.
Table 1 Material parameters for the isotropic and anisotropic models of Sect. $2 \alpha_{1}, \alpha_{2}, \alpha_{\text {elastin }}, \alpha^{(1)}, \alpha^{(2)}\left[\mathrm{dyn} / \mathrm{cm}^{2}\right] ; \gamma_{1}, \gamma_{2}, \gamma_{\text {elastin }}, \gamma^{(1)}$, $\gamma^{(2)}[-] ; \beta^{(1)}, \beta^{(2)}$ [o]

\begin{tabular}{ll}
\hline Model & Parameters \\
\hline Isotropic models & \\
EXP1 & $\alpha_{1}=7.6350 \times 10^{4}$ \\
& $\gamma_{1}=0.7410$ \\
& $\alpha_{2}=6.8220 \times 10^{4}$ \\
EXP2 & $\gamma_{2}=0.0609$ \\
& \\
Anisotropic models & $\alpha_{\text {elastin }}=1.7471 \times 10^{4}$ \\
(EXP1, EXP2-ZS) & $\gamma_{\text {elastin }}=0.8620$ \\
& $\alpha^{(1)}=\alpha^{(2)}=1.4979 \times 10^{5}$ \\
& $\gamma^{(1)}=\gamma^{(2)}=0.5736$ \\
& $\beta^{(1)}=-\beta^{(2)}=56.52^{\circ}$ \\
& $\alpha_{\text {elastin }}=3.5270 \times 10^{4}$ \\
& $\gamma_{\text {elastin }}=0.3424$ \\
& $\alpha^{(1)}=\alpha^{(2)}=1.3370 \times 10^{5}$ \\
& $\gamma^{(1)}=\gamma^{(2)}=0.2141$ \\
& $\beta^{(1)}=-\beta^{(2)}=42.82^{\circ}$ \\
& $\lambda_{r}^{A,(1)}=\lambda_{r}^{A,(2)}=1.5009$ \\
\hline &
\end{tabular}

3.1 Material parameters for the blood flow and arterial tissue models

The material constants for blood density and viscosity are $\rho_{f}=1.000 \mathrm{~g} / \mathrm{cm}^{3}$ and $\mu_{f}=0.035 \mathrm{~g} /(\mathrm{cm} \mathrm{s}$ ) (see e.g. $[30,68])$. Regarding the resistance boundary condition of Eq. (17) on the outflow section of the fluid domain, we set the coefficient as follows: $R=1.032 \times 10^{6}(\mathrm{dyn} \mathrm{s}) / \mathrm{cm}^{5}$ and $p_{V}=0 \mathrm{dyn} / \mathrm{cm}^{2}$.

Since we specifically focus on FSI numerical simulations on cerebral arteries, the values of the material parameters have been taken from [85] where the numerical validation of the constitutive models is presented.

We remark that in Table 1, it is assumed that two families of collagen fibers are immersed in the isotropic elastin network [85]. In addition, both families have the same mechanical properties, as already done in e.g. [32,50]. In references $[50,51,85]$ the angle $\beta^{(i)}$ formed by the local alignment vector of the $i$-th fibers family in the unloaded reference configuration (see Fig. 2) and the circumferential direction of the vessel and the circumferential deformation $\lambda_{r}^{A,(i)}$ at which the collagen fibers activation occurs for the anisotropic (EXP1, EXP2-FS) model are determined by means of the leastsquares approximation of the experimental measurements of [73]. Indeed, experimental observations of the spatial orientation of the collagen fibers and of their activation stretch are not reported in [73]. The fitted stress-strain relations obtained for the different constitutive models are represented in Fig. 3. 


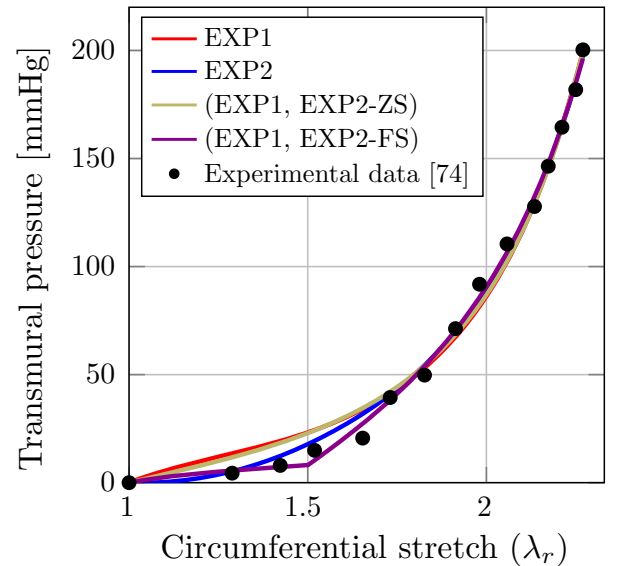

Fig. 3 Least-squares approximation of the experimental data of [73] for the constitutive models of Sect. 2 and the material parameters reported in Table 1 [Picture from [85]]

In addition, for the numerical simulations of Sect. 3.3, we set $\kappa=9.0 \times 10^{6} \mathrm{dyn} / \mathrm{cm}^{2}$ in Eq. (8) such that the incompressibility constraint $J=1$ is nearly full filled with a margin of $1 \%$ for all physiological deformations considered in this work. In order to achieve physiological displacements of the vessel wall we set the elastic coefficient $K$ in the external Robin boundary condition of Eq. (18) as $K=10^{7} \mathrm{dyn} / \mathrm{cm}^{3}$.

\subsection{Space and time discretization of the coupled FSI problem}

The coupled FSI problem presented in Sect. 2 has been discretized in space by means of the finite element method [65]. More in detail, the Navier-Stokes equations have been discretized by means of the Inf-Sup stable pair of finite element spaces of functions $\mathbb{P} 1$ Bubble- $\mathbb{P} 1$ for the velocity and pressure, respectively, and linear finite elements $(\mathbb{P} 1)$ discretize both the solid and geometric problems. The NavierStokes and structure equations have been discretized in time by means of a second order Backward Differentiation Formula scheme. The discrete coupled FSI system is solved by means of the so called Fully Implicit monolithic technique, which is a direct coupling technique [20]. As detailed in [20], according to the Fully Implicit monolithic technique, all the nonlinear terms in the fluid and solid equations as well as the boundary terms that depend on the solution, i.e. the outflow $Q(\boldsymbol{u})$ in Eq. (17) and the Robin boundary condition on $\mathcal{B}_{0, \text { ext }}$ are discretized implicitly in time; this choice leads to a more accurate and robust time discretization of the FSI problem compared to the one obtained by means of other strategies, such as the geometric-convective explicit (GCE) [20] for which the advection nonlinear term, depending on the fluid and ALE velocities $\boldsymbol{u}$ and $\boldsymbol{w}_{\mathcal{F}}$, in the Navier-Stokes equations is linearized. In the Fully Implicit technique, the resulting nonlinear system is solved iteratively by the New-

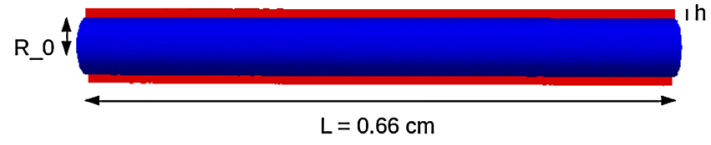

(a) Straight cylinder

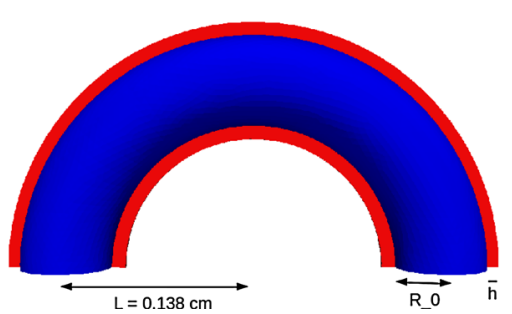

(b) Toroidal geometry

Fig. 4 Idealized geometries of blood vessels. The internal radius $R_{0}$ and wall thickness $h$ represent the characteristic dimensions of the anterior cerebral artery [73]

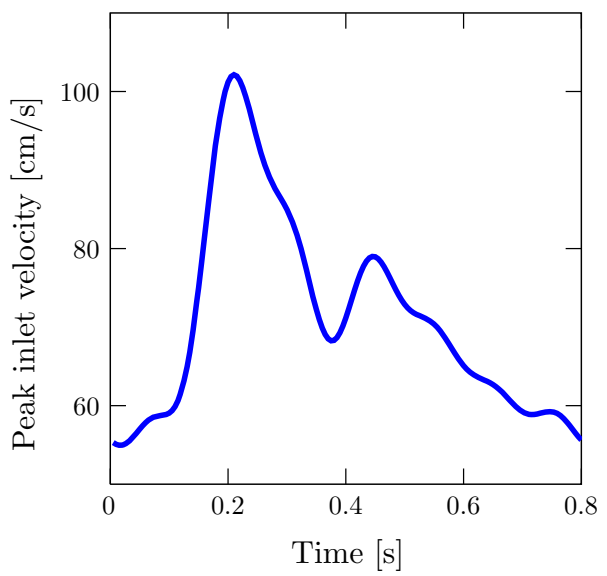

Fig. 5 Peak velocity of the inlet parabolic profile $\boldsymbol{u}_{\text {inlet }}$ imposed on the inlet boundary of the fluid domain $\left.\mathcal{F}(t)\right|_{\text {inlet }}$

ton method [63]. At each iteration, the coupled FSI problem is linearized with respect to the fluid, solid, and geometric unknowns and the resulting linear system is solved by means of the GMRES method [71] using an Additive-Schwarz preconditioner [64]. We report the total number of degrees of freedom of the FSI monolithic system case by case in the next sections.

The numerical simulations have been carried out in computational domains whose characteristic dimensions reproduce the physiological ones of the anterior cerebral artery. For this reason, we consider a cylindrical geometry of blood vessel with internal undeformed radius $R_{0}=0.033 \mathrm{~cm}$ (see [89]) and wall thickness $h=0.01 \mathrm{~cm}$, as in [73]. Furthermore, we take into account a toroidal geometry of artery whose internal radius and thickness are the same as before; see Fig. 4. In addition, Fig. 5 reports the time evolution of the rescaled peak inlet velocity for the inlet boundary conditions of Eq. (16) [2]. 


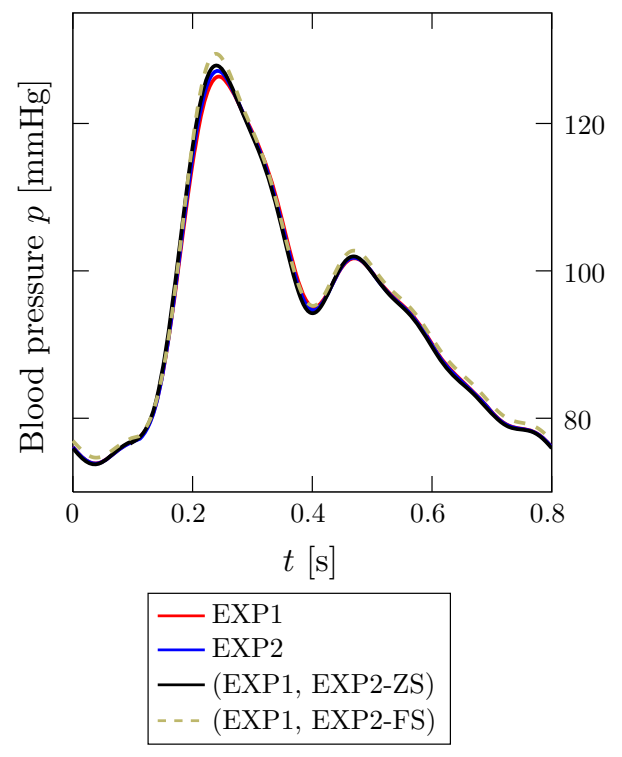

Fig. 6 Blood pressure $p$ versus time at the cross section $z=0.33 \mathrm{~cm}$ for the different constitutive models

\subsection{Numerical results}

\subsubsection{Straight cylindrical geometry}

The fluid and structure meshes under consideration are composed of 156,593 and 113,880 tetrahedral elements, respectively, yielding a total number of degrees of freedom of the monolithic system equal to 747,410 . In order to analyze the numerical results, two complete heart beats have been numerically simulated for all the cases under consideration.

Figure 6 shows the time evolution of the mean blood pressure at the cross section $z=L / 2=0.33 \mathrm{~cm}$ along the centerline, measured for all the constitutive material models employed. We remark the experimental measurements of blood pressure $p$ in cerebral arteries reported in $[26,38]$ indicate the strong pulsatile qualitative behavior of $p$ during the cardiac cycle, as obtained in this work. In addition, we report that the values of $p$ obtained during the heart beat are quantitatively consistent with the ones presented in $[81,86]$ for physiological numerical simulations of cerebral circulation. Furthermore, the time evolution of the mean blood pressure at $z=0.33 \mathrm{~cm}$ is very similar for all the numerical simulations performed with the different constitutive models, which suggests that in this configuration, the modeling choice for the arterial wall does not strongly affect the hemodynamics inside the vessel lumen and vice-versa. Figure 7 presents the blood pressure and velocity in the deformed fluid domain at the time $t=0.60 \mathrm{~s}$, i.e. at the diastolic phase. Since similar results were obtained with all the constitutive laws, only the spatial distribution of the fluid pressure $p$ in the case of the EXP1 model for the vessel wall is shown. In Fig. 7a we highlight the small spatial gradient of the pressure field $p$

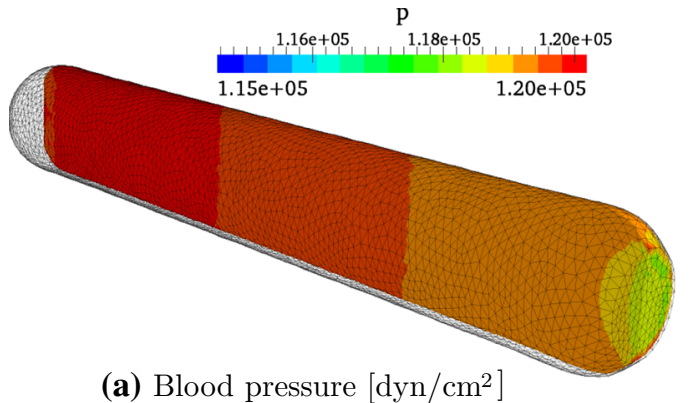

(a) Blood pressure $\left[\mathrm{dyn} / \mathrm{cm}^{2}\right]$

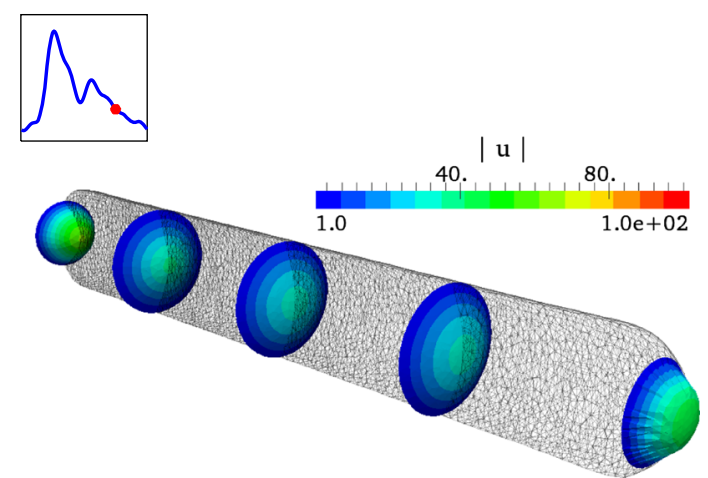

(b) Velocity magnitude $[\mathrm{cm} / \mathrm{s}]$

Fig. 7 Blood pressure and velocity magnitude at the time $t=0.60 \mathrm{~s}$; in $\mathbf{b}$ each cross section is deformed according to the vectorial field $\boldsymbol{u}$

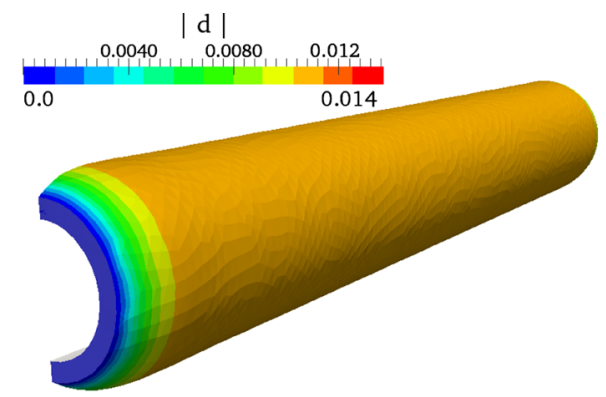

(a) External view
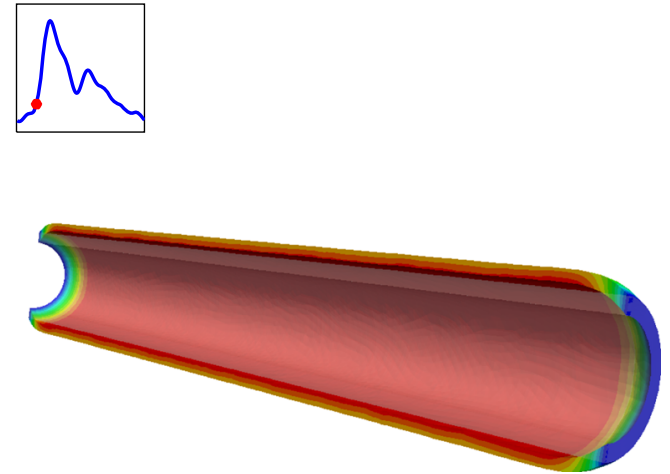

(b) Internal View

Fig. 8 Displacement magnitude $(\mathrm{cm})$ on the deformed vessel wall. a External view; b internal view. Time $t=0.100 \mathrm{~s}$. Constitutive model EXP2. The gray overlay represents the undeformed domain 


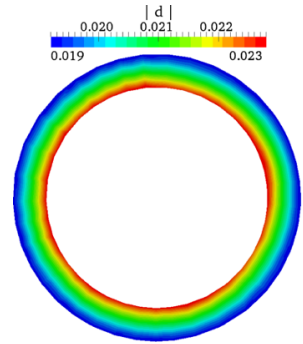

(a) EXP1

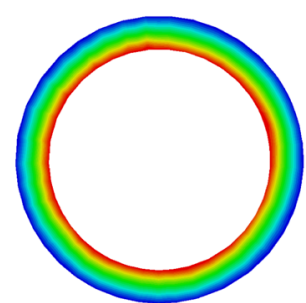

(c) (EXP1, EXP2-ZS)

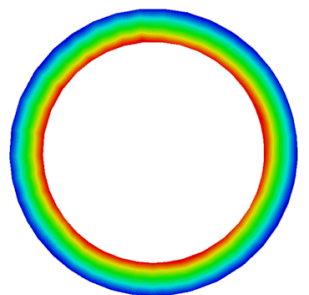

(b) EXP2

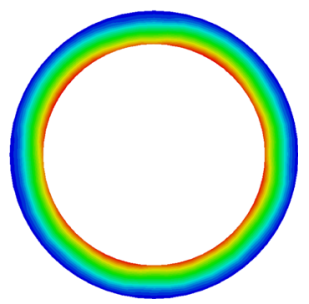

(d) (EXP1, EXP2-FS)
Fig. 9 Displacement $\widehat{\boldsymbol{d}}(\mathrm{cm})$ of the vessel wall at the cross section $z=0.33 \mathrm{~cm}$ for the different constitutive models at the systolic peak at time $t=0.212 \mathrm{~s}$

along the axis of the cylindrical geometry due to the resistance condition considered at the outlet surface of the fluid domain. At the same time, in Fig. 7b, we report that the fluid velocity profile is mostly parabolic at all the cross sections considered.

Figure 8 shows deformed the vessel wall at the time $t=0.10 \mathrm{~s}$. As for the fluid velocity and pressure, similar results were obtained for all the models; therefore, the deformations of the solid domain are presented only in the case of the isotropic EXP2 law. We remark that during the heart beat, the vessel wall deforms mainly in the radial direction rather than along the axial direction of the domain. In addition, the characteristic propagation of the pressure wave from the inlet towards the outlet of the fluid domain, which is often obtained within the context of FSI numerical simulations on idealized geometries of vessels (see e.g. [27,30,47]), is not observed in this case due to the combined effects of the resistance and Robin boundary conditions on the outlet and external surfaces of the fluid and solid domains, respectively. We remark that the radial deformations reported in Fig. 8 are maintained during the whole cardiac cycle, as also presented in Fig. 9 corresponding to the systolic peak of the inlet profile. Figure 10 presents the radial displacement at one point of the internal surface of the vessel wall during the cardiac cycle. In Fig. 10, for each constitutive model, the maximum and minimum values of the radial displacement indicate a change in the vessel radius of approximately $27 \%$ with respect to the undeformed internal radius $R_{0}$ during the heart beat. This value is consistent with the experimental measurements reported in [34] for the cerebral vasculature. In addition, Fig. 10 shows that the choice of the constitutive model for the arterial tissue

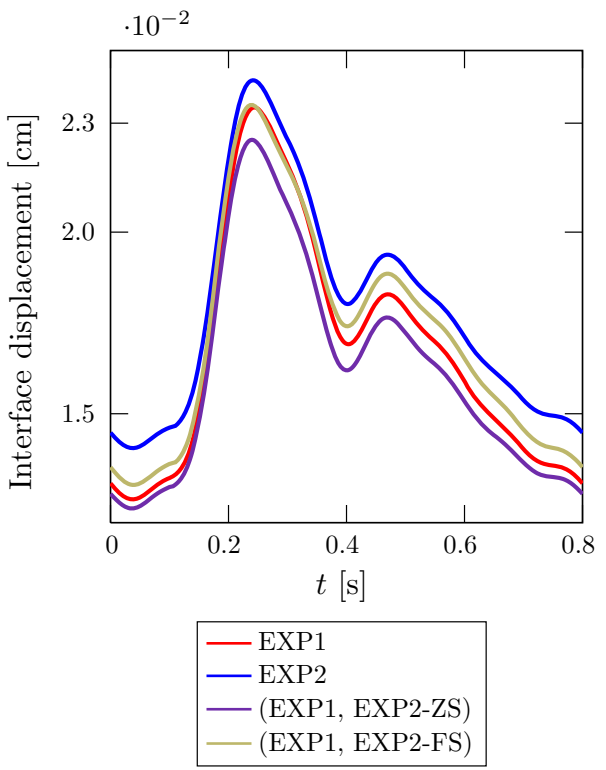

Fig. 10 Displacement $(\mathrm{cm})$ at one point of the fluid-structure interface at the cross section $z=0.33 \mathrm{~cm}$ during the heart beat for the different constitutive models

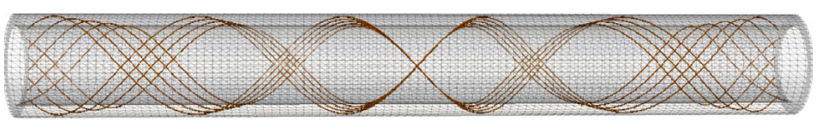

(a) Collagen fibers in the vessel wall

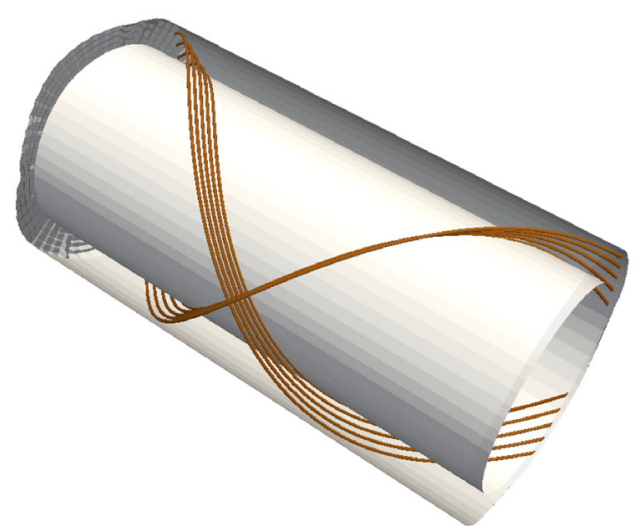

(b) Zoom in a central portion

Fig. 11 Representation of the collagen fibers in the reference configuration $\mathcal{B}_{0}$ of the arterial wall. Constitutive model: (EXP1, EXP2-FS), see Table 1

substantially affects the resulting vessel wall displacement. More specifically, among the isotropic models, the smallest displacements are obtained with the EXP1 law, whereas the highest displacements with the EXP2 law; more precisely, the displacement predicted by the EXP2 model is around 6\% higher than the one obtained with the EXP1 model during the heart beat. We recall that the material parameters were obtained in [85] from fitting experimental data on cerebral arteries. The deformation of the vessel wall depends on the 


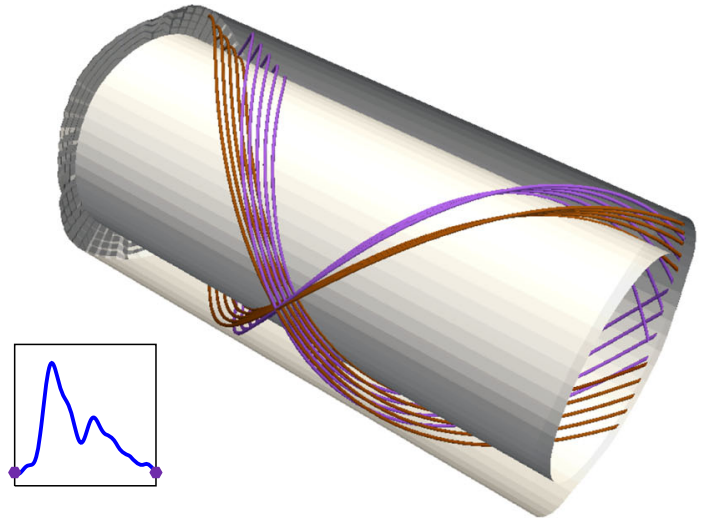

(a) Deformed (purple) collagen fibers at the beginning and end of the heart beat

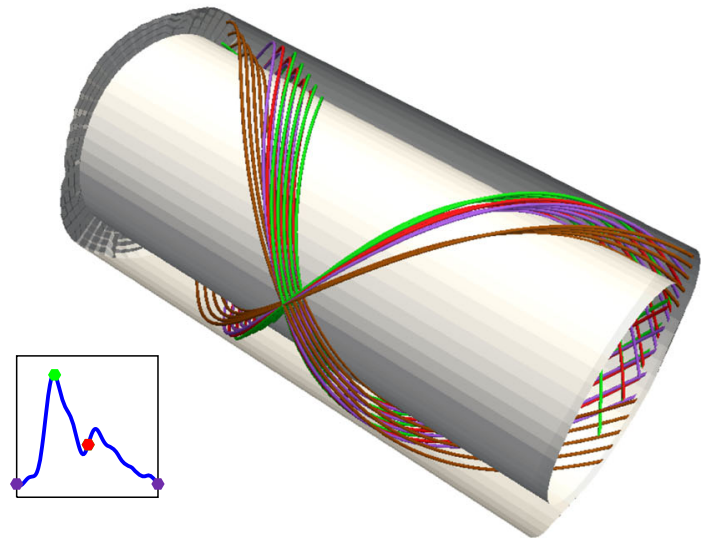

(b) Deformation of the collagen fibers at different times of the heart beat

Fig. 12 Representation of the deformed collagen fibers at different times during the heart beat. Constitutive model: (EXP1, EXP2-FS). The brown colored lines represent the collagen fibers in the undeformed configuration $\mathcal{B}_{0}$ of the vessel wall; $\left|\beta^{(1)}\right|=\left|\beta^{(2)}\right|=0.7473 \mathrm{rad}$

transmural pressure $(\Delta P)$ which is the difference between the pressure acting on the wall by the blood and the external pressure load on the wall. The latter is determined by the Robin boundary condition. In these simulations, the variation in transmural pressure varies between 3 and $15 \mathrm{mmHg}$. As discussed in Sect. 3.1, for this range of transmural pressures and for the material parameters of Table 1, the isotropic EXP1 and EXP2 models represent the most and the least rigid material, respectively (see Fig. 3); similarly, for the differences in the numerical results among the two anisotropic models, see Fig. 3.

When anisotropic constitutive laws for the vessel wall are employed, it is interesting to investigate the deformations of the collagen fibers during the heart beat to estimate their contribution to the overall mechanical response of the vessel wall. In order to qualitatively highlight the role of the collagen fibers orientation during the heart beat, the (EXP1, EXP2-FS) model is considered; however, a similar result has

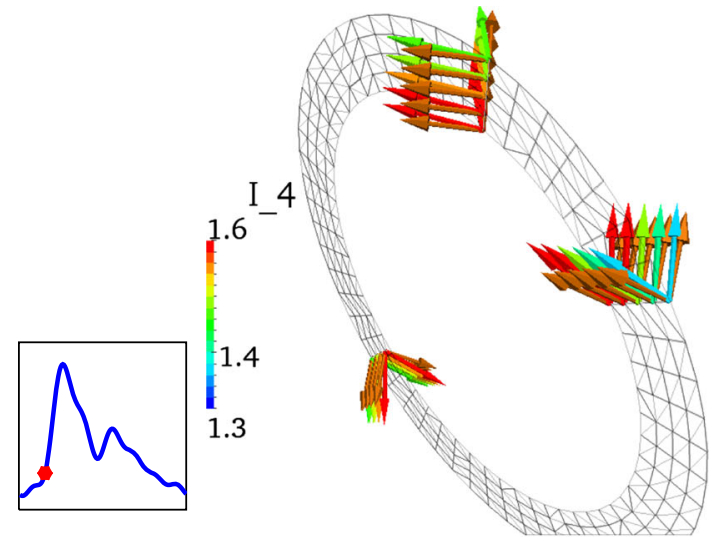

(a) $t=0.100 \mathrm{~s}$

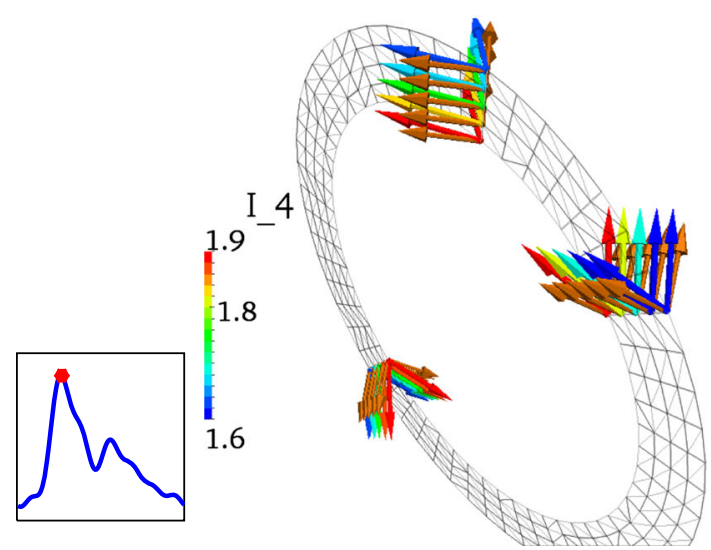

(b) $t=0.212 \mathrm{~s}$

Fig. 13 Local alignment of the collagen fibers at the cross section $z=0.33 \mathrm{~cm}$. Constitutive model: (EXP1, EXP2-FS). The brown arrows represent the local alignment of collagen fibers in the undeformed configuration $\mathcal{B}_{0}$ of the vessel wall; $\left|\beta^{(1)}\right|=\left|\beta^{(2)}\right|=42.82^{\circ}$

been observed also for the (EXP1, EXP2-ZS) model. Figure 11 shows the two families of fibers passing through the points lying on the intersection between the vertical plane $z=0.33 \mathrm{~cm}$ and the axis $y=0$. As reported in Tab. 1, the characteristic angle $\beta^{(i)}$ in the reference configuration $\mathcal{B}_{0}$, for $i=1,2$, between the local alignment of each family and the circumferential direction is $\left|\beta^{1}\right|=\left|\beta^{2}\right|=41.82^{\circ}$. In Fig. 11, the collagen fibers are presented in their undeformed state, i.e. for zero displacement of the vessel wall. On the other hand, Fig. 12 shows the deformations of the collagen fibers during the heart beat. The variation of their local direction is highlighted in Figs. 12 and 13; the angle between the local alignment of the single family of collagen fibers and the circumferential direction decreases as the radial displacement increases. In particular, the angle between the deformed vector representing the deformed local alignment of the single family of fibers and the circumferential direction at the beginning of the heart beat is indicated by $\beta_{H B}^{(i)}$ and it is $\left|\beta_{H B}^{(1)}\right|=\left|\beta_{H B}^{(2)}\right|=35.70^{\circ}$. As expected, the maximum variation in the local alignment of the collagen fibers in the heart 


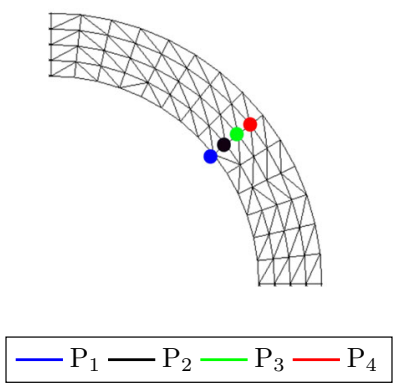

(a) Points locations

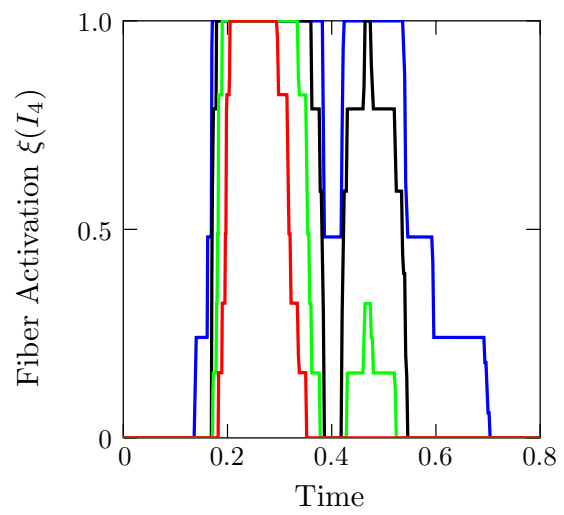

(c) Collagen fibers activation

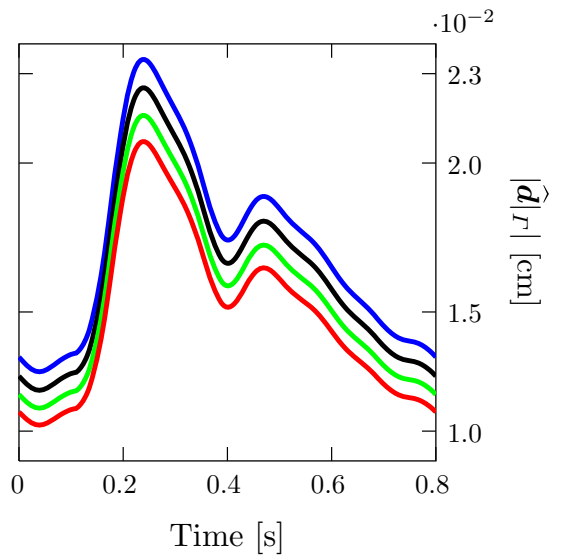

(b) Displacement magnitude at each point

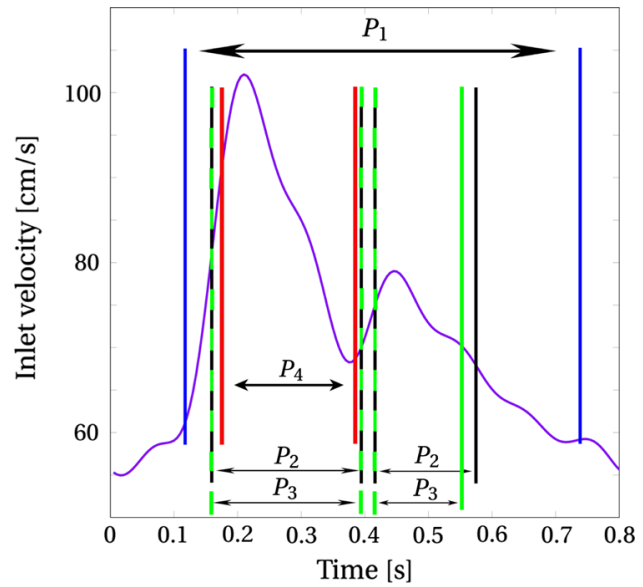

(d) Collagen fibers activation with respect to the inlet blood flow velocity

Fig. 14 Displacement and activation parameters and times through the thickness of the vessel wall for the anisotropic model (EXP1, EXP2-FS)

beat with respect to $\beta_{H B}^{(1)}$ is observed corresponding to the systolic peak of the inlet velocity profile, for which the measured angle is $\left|\beta_{\text {systole }}^{(1)}\right|=\left|\beta_{\text {systole }}^{(2)}\right|=33.70^{\circ}$. Therefore, in the case of the (EXP1, EXP2-FS) model, for physiological diastolic and systolic blood pressures, the change in the local orientation of the collagen fibers is about $7 \%$ with respect to $\beta_{H B}^{(1)}$. As already mentioned, qualitatively similar results have been obtained in the case of the (EXP1, EXP2-ZS) model for which $\left|\beta^{1}\right|=\left|\beta^{2}\right|=56.52^{\circ},\left|\beta_{H B}^{(1)}\right|=\left|\beta_{H B}^{(2)}\right|=51.36^{\circ}$, and $\left|\beta_{\text {systole }}^{(1)}\right|=\left|\beta_{\text {systole }}^{(2)}\right|=47.17^{\circ}$; therefore the maximum variation is about $8 \%$ with respect to $\left|\beta_{H B}^{(1)}\right|$. On the other hand, the variation between $\beta^{(1)}$, which is provided in $\mathcal{B}_{0}$ by means of the data fitting, and $\beta_{H B}^{(1)}$ (and, similarly, $\beta^{(2)}$ and $\left.\beta_{H B}^{(2)}\right)$ is affected by the diastolic pressure attained in the numerical simulations. As presented in Fig. 13, the largest stretch of the collagen fibers occurs at the internal surface of the vessel wall in correspondance with the largest displacement. It is worth pointing out that the local orientations of the two families of fibers remain symmetric with respect to the circumferential direction of the cylinder since, as presented in Figs. 8 and 9, the vessel wall deforms along the radial direction.

Finally, we are interested in understanding the progressive activation and deactivation of the collagen fibers through the thickness of the vessel wall when the anisotropic (EXP1, EXP2-FS) model is employed. We show the time evolution of the mechanical activation of the collagen fibers through the thickness of the arterial wall in Fig. 14a for four points along the radial direction of the domain at $z=0.33 \mathrm{~cm}$. Figure $14 \mathrm{~b}, \mathrm{c}$ present the displacement and the mechanical activation of the collagen fibers for each of the four points. As the arterial tissue displacement decreases moving along the radial direction from the internal toward the external surface of the domain, analogously the total amount of time for which the collagen fibers are active during the heart beat decreases. We remark that the qualitative piecewise constant behavior in time of the activation functions in Fig. 14c is due 
Internal view

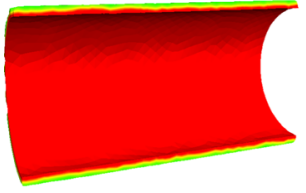

(a) EXP1

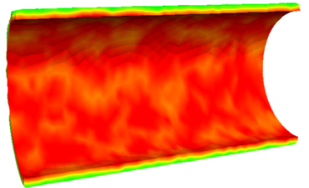

(c) $(\mathrm{EXP} 1, \mathrm{EXP} 2-\mathrm{ZS})$

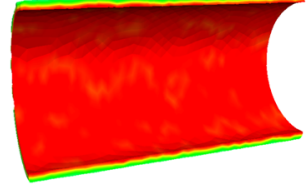

(b) EXP2

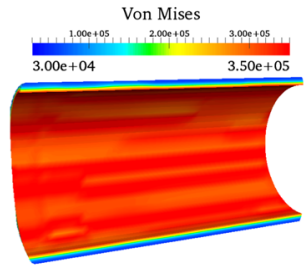

(d) (EXP1, EXP2-FS)

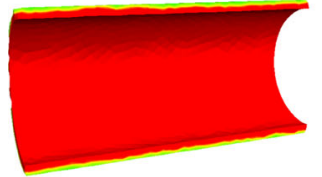

(a) EXP1

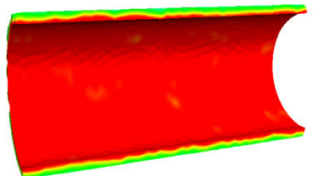

(c) $(\mathrm{EXP} 1, \mathrm{EXP} 2-\mathrm{ZS})$
Internal view

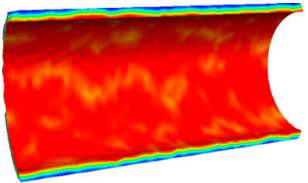

(b) EXP2

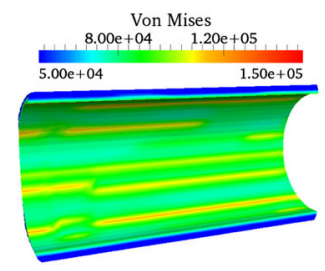

(d) (EXP1, EXP2-FS)
External view

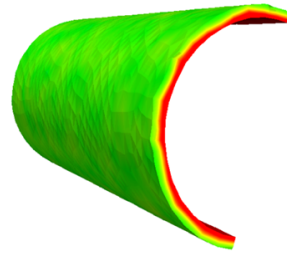

(a) EXP1

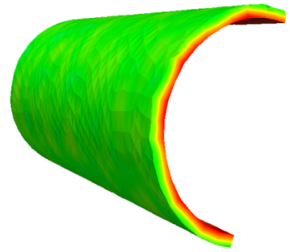

(c) $(\mathrm{EXP} 1, \mathrm{EXP} 2-\mathrm{ZS})$
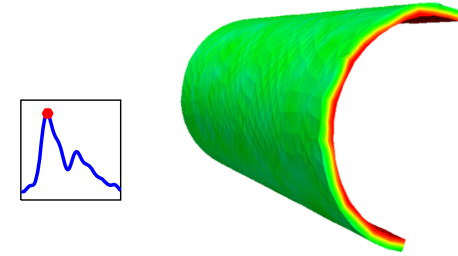

(b) EXP2

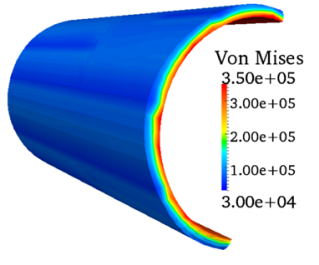

(d) (EXP1, EXP2-FS)
Fig. 15 Von Mises stress $\left(\mathrm{dyn} / \mathrm{cm}^{2}\right)$ for the different constitutive models at the time $t=0.212 \mathrm{~s}$. Internal view (top); external view (bottom)

to the high value of the activation parameter $\epsilon=5.0 \times 10^{5}$ in Eq. (15) which leads to a highly steep activation function. Figure $14 d$ reports the activation time intervals for each of the four points presented in Fig. 14a in comparison with the physiological inlet velocity profile. It can be observed that at the innermost point $P_{1}$, the collagen fibers are mechanically active for almost the entire duration of the heart beat, while at $P_{4}$, the outermost point under consideration, the collagen fibers activation occurs only during the systolic peak. On the other hand, at the points $P_{2}$ and $P_{3}$, which are internal to the arterial wall, the collagen fibers actively contribute to the mechanical response of the tissue during both the systolic and the diastolic phases of the heart beat even if not in a continuous manner. This numerical result indicates the relevance of modeling the recruitment of the collagen fibers at finite strains in order to highlight the protective role of the fibers in the outermost layers of the arterial tissue. On the other hand, with the (EXP1, EXP2-ZS) model, we recall that

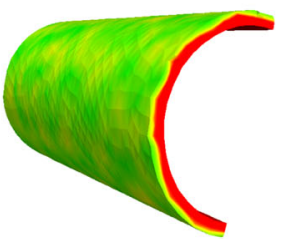

(a) EXP1

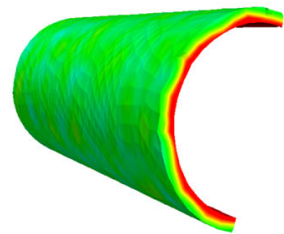

(c) $(\mathrm{EXP} 1, \mathrm{EXP} 2-\mathrm{ZS})$
Internal view

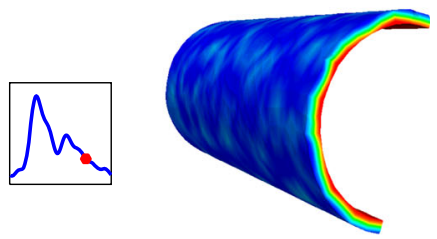

(b) EXP2

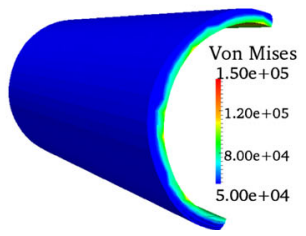

(d) $(\mathrm{EXP} 1, \mathrm{EXP} 2-\mathrm{FS})$
Fig. 16 Von Mises stress $\left(\mathrm{dyn} / \mathrm{cm}^{2}\right)$ for the different constitutive models at the time $t=0.600 \mathrm{~s}$. Internal view (top); external view (bottom)

the collagen fibers are always mechanically active during the heart beat; therefore, their progressive activation cannot be captured when considering such constitutive law.

Figures 15 and 16 show the spatial distribution of the Von Mises stress $\left(\sigma_{V M}\right)$ [41] obtained with the different constitutive models at different times of the heart beat in a central portion of the solid domain. We observe that the two isotropic models and the (EXP1, EXP2-ZS) law predict similar values of $\sigma_{V M}$ during the systolic phase, as represented in Fig. 15, while smaller values of $\sigma_{V M}$ stress result from in the case of the (EXP1, EXP2-FS) model, especially at the outer surface of the arterial wall. In particular, we highlight that in Fig. 15, although the values of Von Mises stress reached on the internal surface of the vessel wall are similar, the spatial distribution of $\sigma_{V M}$ is strongly affected by the choice of the constitutive model. Similarly, the constitutive model for the vessel wall affects the values of $\sigma_{V M}$ measured along the thickness of the arterial tissue during 


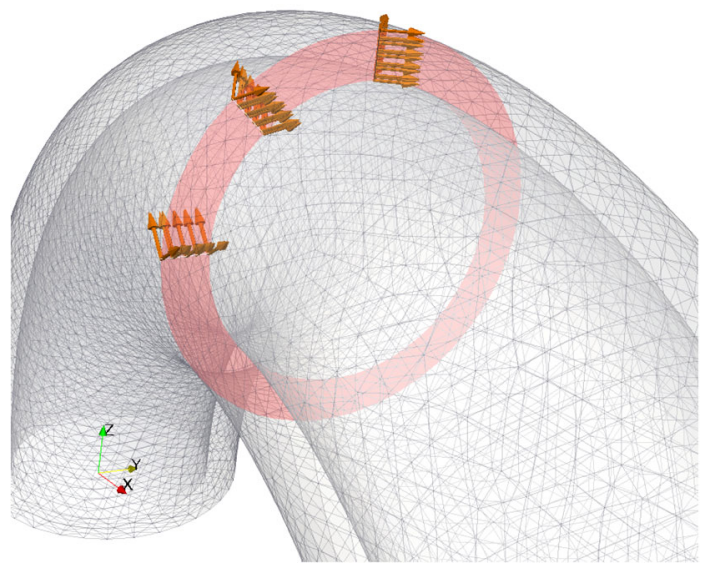

(a) Case $\mathrm{A}$

$\left(\beta^{(1)}=-\beta^{(2)}=56.52^{\circ}\right)$

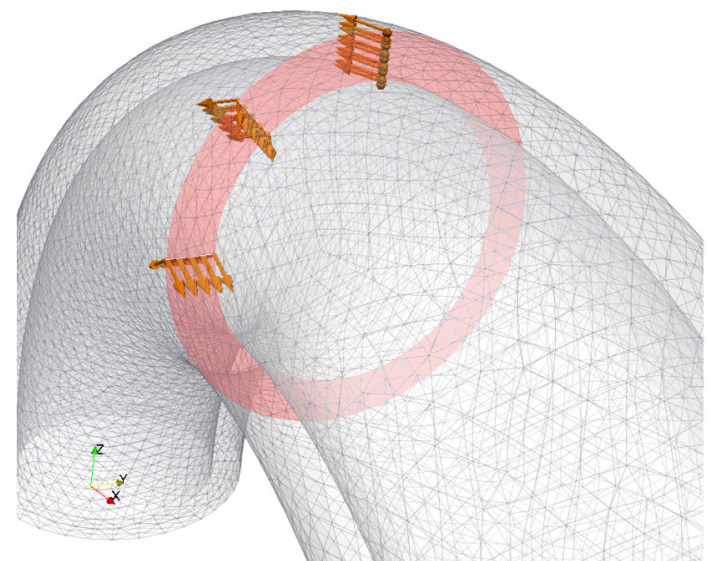

(b) Case B

$\left(\beta^{(1)}=-\beta^{(2)}=180^{\circ}-56.52^{\circ}\right)$

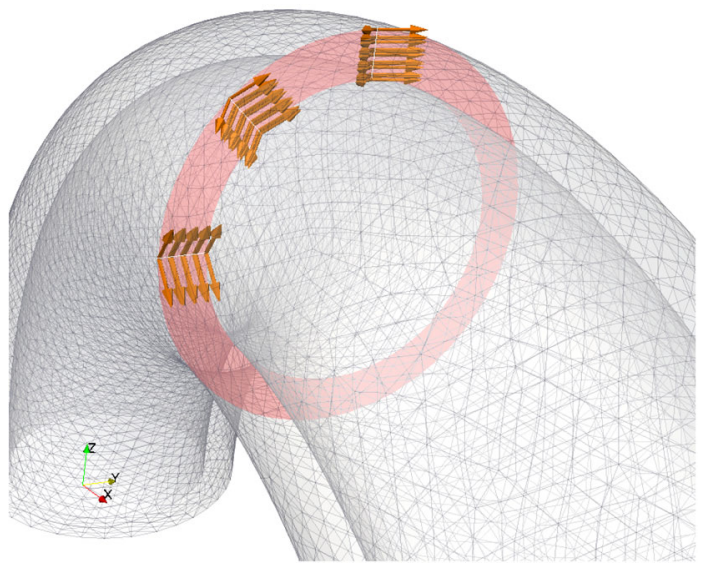

(c) Case $\mathrm{C}$

$\left(\beta^{(1)}=-\beta^{(2)}=90^{\circ}+56.52^{\circ}\right)$

Fig. 17 Vectorial representation of the two families of fibers in the (EXP1, EXP2-ZS) model on one cross section of the solid domain for the sensitivity study on the vessel wall displacement with respect to the collagen fibers orientation

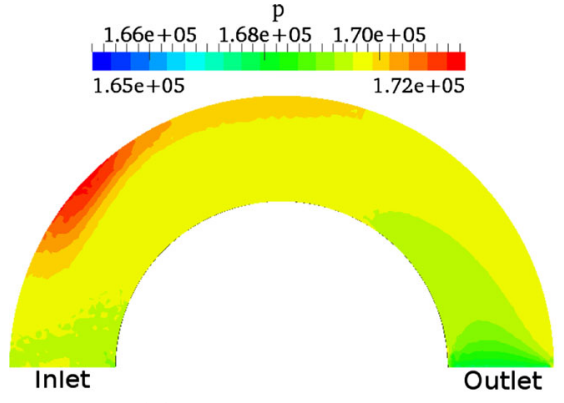

(a) Blood pressure

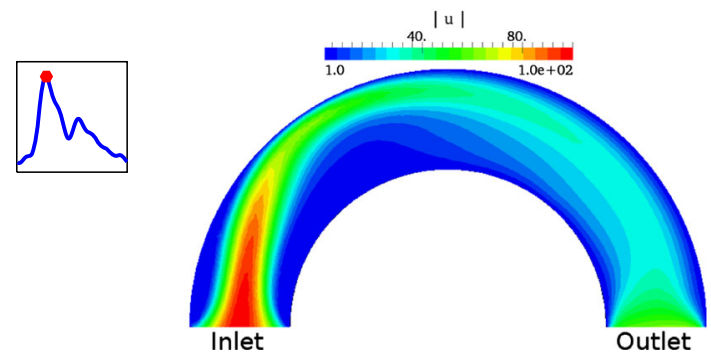

(b) Blood velocity

Fig. 18 Blood pressure $\left.p\left(\mathrm{dyn} / \mathrm{cm}^{2}\right]\right)$ and velocity $(\mathrm{cm} / \mathrm{s})$ in the undeformed fluid domain at the time $t=0.212 \mathrm{~s}$. Constitutive model for the arterial wall: EXP2

the diastolic phase of the cardiac cycle, as represented in Fig. 16. In addition, while the anisotropic model with collagen recruitment at finite strains still yields the lowest values of Von Mises stress also during the diastolic phase, significant differences in $\sigma_{V M}$ obtained with the two isotropic laws and the anisotropic (EXP1, EXP2-ZS) model, as observed in Fig. 16. We remark that the results reported in Figs. 15 and 16 are consistent with the stress-strain relations reported in Fig. 3 for which the EXP1 model and the (EXP1, EXP2-FS) represent the most and the least rigid materials, respectively, among the ones under consideration.

\subsubsection{Toroidal geometry}

We carry out numerical simulations of the FSI problem for a toroidal geometry of the blood vessel represented in Fig. 4b. The same material parameters for the arterial wall models presented in Table 1 are employed. In order to reproduce the same physiological blood flow, pressures, and arterial wall displacement during the heart beat, the same set of boundary conditions described in Sect. 2.3 is considered. The fluid and solid meshes are composed of 176,641 and 106,212 linear tetrahedral elements, respectively; the finite elements spaces for the fluid, solid and geometric problems are the same as in Sect. 3.3.1 for which the total number of DOFs of the FSI monolithic system is 822,695 . In order to reduce the number of cases under consideration, due to the similarities that this case presents with respect to the one described in 

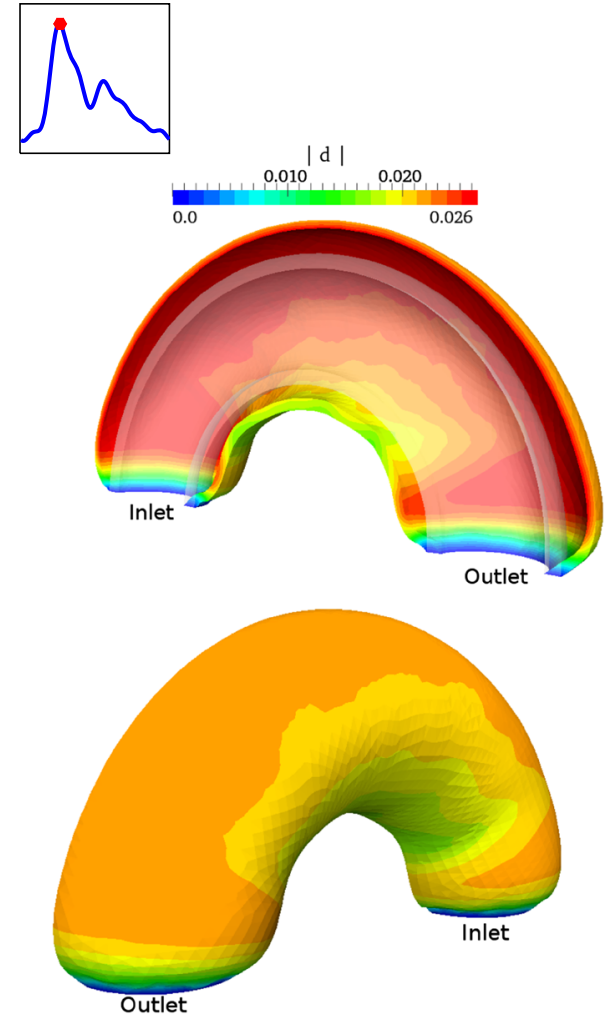

(a) $\mathrm{t}=0.212 \mathrm{~s}$
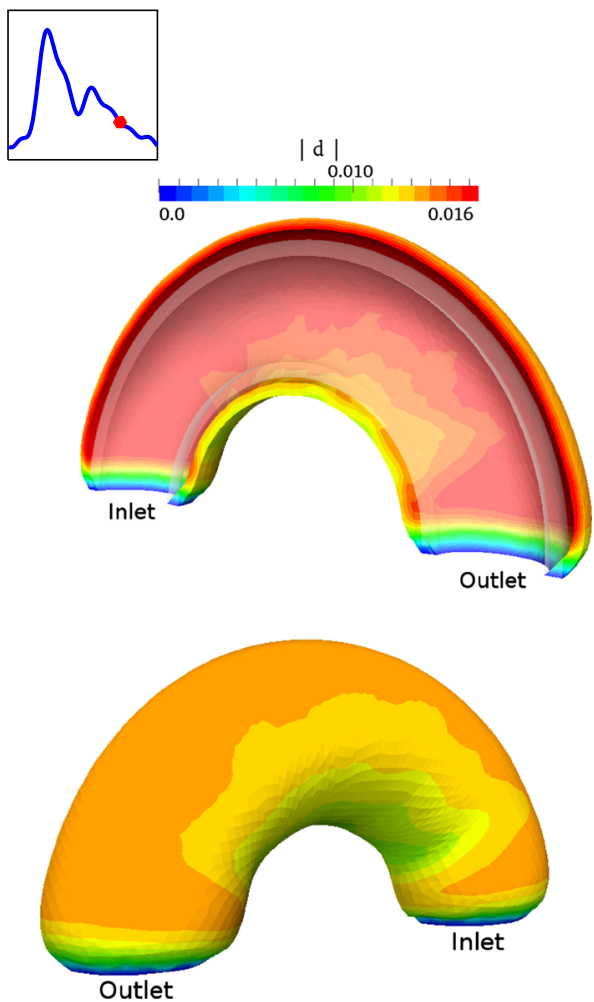

(b) $\mathrm{t}=0.60 \mathrm{~s}$

Fig. 19 Magnitude of the vessel wall displacement $\widehat{\boldsymbol{d}}(\mathrm{cm})$ in the deformed fluid domain. Constitutive model for the arterial wall: EXP2. In the first row, the gray overlay represents the undeformed domain

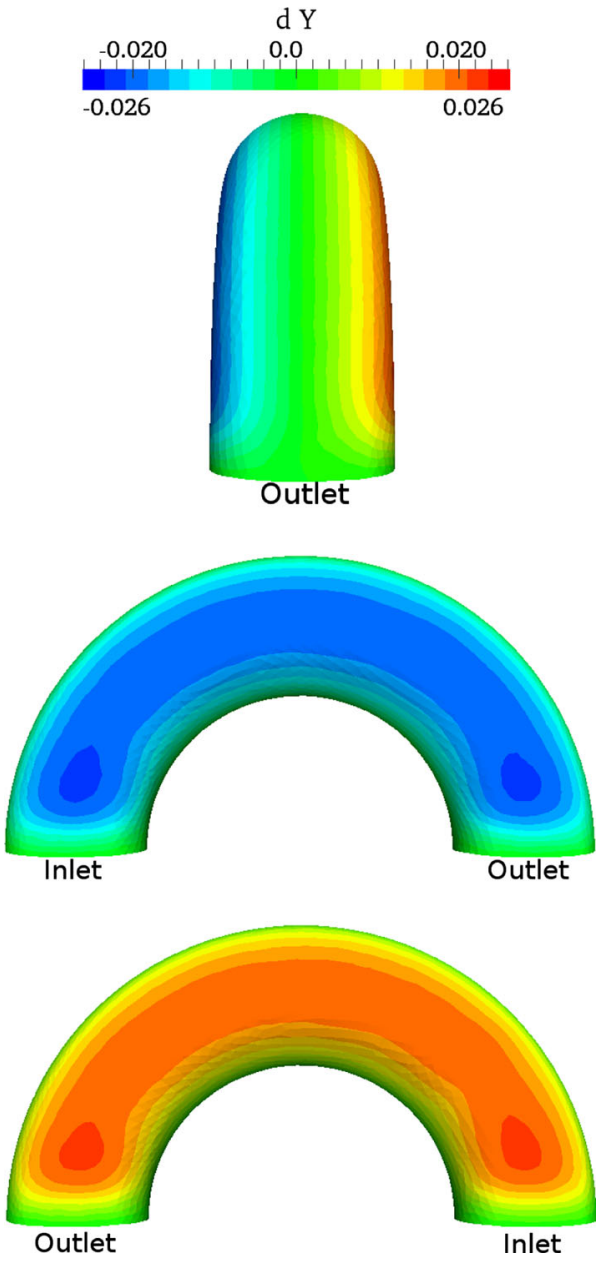

Fig. 20 Component along the $y$ axis of the arterial wall displacement $\widehat{d}(\mathrm{~cm})$ at the systolic peak, $t=0.212 \mathrm{~s}$. Constitutive model: EXP2

Sect. 3.3.1, the FSI coupled system has been simulated only in the cases of the isotropic EXP2 and the anisotropic (EXP1, EXP2-ZS) laws. ${ }^{1}$ In the case of the (EXP1, EXP2-ZS), it is assumed that the characteristic direction of the two families of collagen fibers forms with the main radial direction of the torus the angle is $\left|\beta^{(1)}\right|=\left|\beta^{(2)}\right|=56.52^{\circ}$ as in Sect. 3.3.1, see Fig. 17a.

Figure 18 shows the blood pressure and velocity at the systolic peak of the cardiac cycle at the time $t=0.212 \mathrm{~s}$. We remark that, similarly to what has been reported for the straight cylinder, the minimum diastolic pressure is 75 $\mathrm{mmHg}$, while the maximum systolic pressure is $130 \mathrm{mmHg}$. As represented in Fig. 18, during the heart beat, the maximum blood pressure occurs inside the fluid domain.

1 We remark that the numerical solution of the FSI problem with the (EXP1, EXP2-FS) model is numerically challenging since it requires a suitable tuning and setting of the numerical parameters and discretization in order to be efficient. 


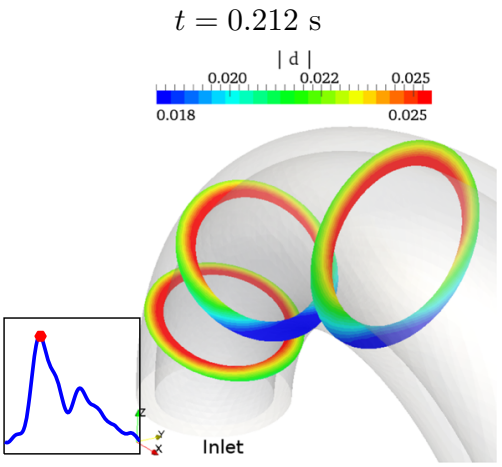

(a) EXP2

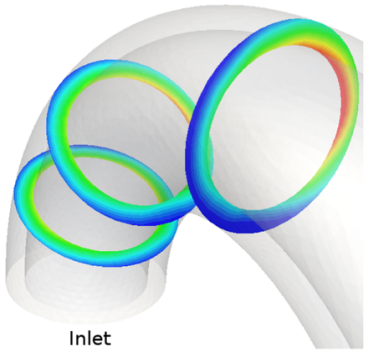

(b) (EXP1, EXP2-ZS)

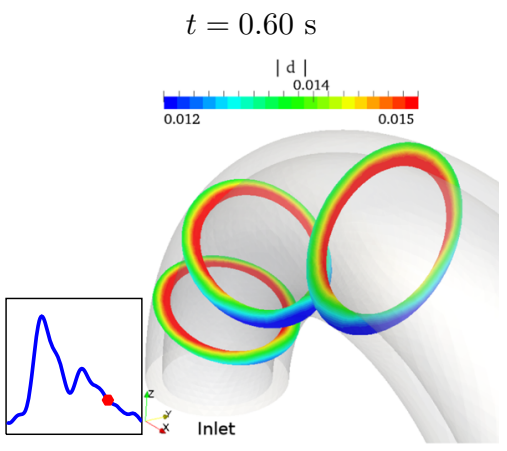

(a) EXP2

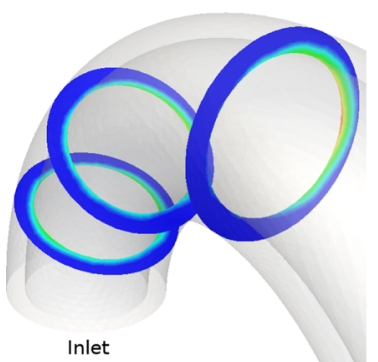

(b) (EXP1, EXP2-ZS)

Fig. 21 Magnitude of the vessel wall displacement $\widehat{\boldsymbol{d}}[\mathrm{cm}]$ on three different radial sections of the vessel wall for the EXP2 and (EXP1, EXP2-ZS) models at different times along the heart beat. $t=0.212 \mathrm{~s}$ (top); $t=0.600 \mathrm{~s}$ (bottom)

As reported in Sect. 3.3.1, due to the external Robin boundary conditions, the vessel wall displacement measured during the heart beat occurs mainly along the radial direction in the local toroidal set of coordinates; see Fig. 19. As

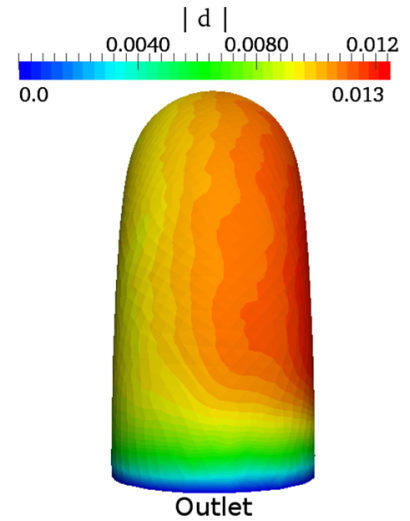

$|\mathrm{d}|$
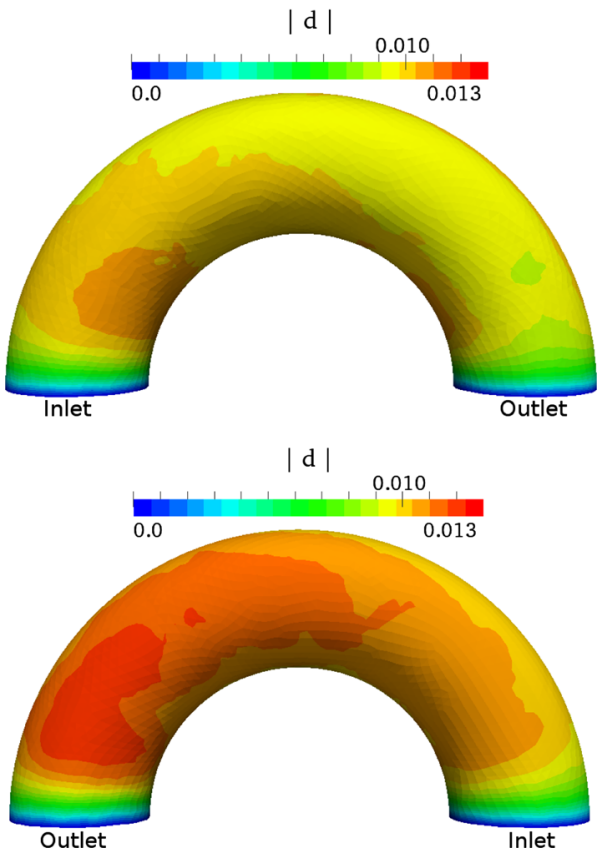

Fig. 22 Vessel wall displacement in the case of the (EXP1, EXP2-ZS) model for the study Case A. Different views. Time $t=0.100 \mathrm{~s}$

it can be noticed, the displacement magnitude is consistent with the one reported in Fig. 9f for the case of the straight cylinder. In particular, for the isotropic EXP2 model, the displacement of the blood vessel is symmetric with respect to a cut along the main circumferential direction of the torus (see Fig. 20); the same type of symmetry was observed in [27] where an isotropic linear elastic constitutive model was employed. On the other hand, the use of the anisotropic model (EXP1, EXP2-ZS) leads to an asymmetric vessel wall displacements during the heart beat. This phenomenon is highlighted in Fig. 21 at two different times of the heart beat. As it can be noticed, although the displacement measured in the case of the (EXP1, EXP2-ZS) law is lower than the one obtained with the EXP2 model, the vessel wall displacement is strongly affected by the presence of the collagenous component in the arterial tissue. In particular, as reported in Figs. 21, 22, 23 and 24, the asymmetry in the arterial wall dis- 


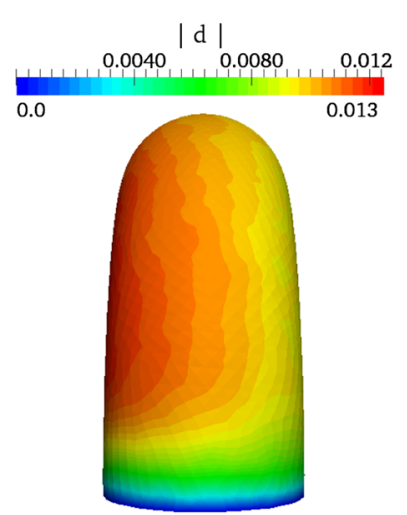

$|\mathrm{d}|$

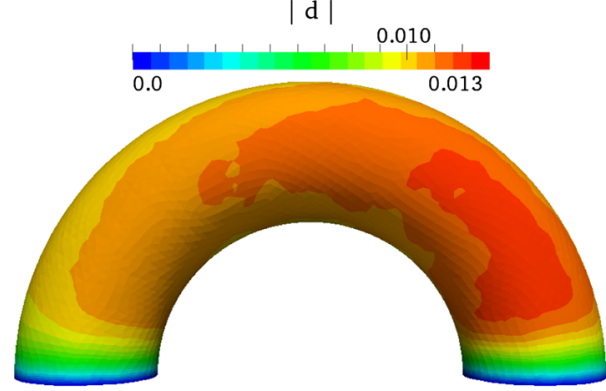

$|\mathrm{d}|$

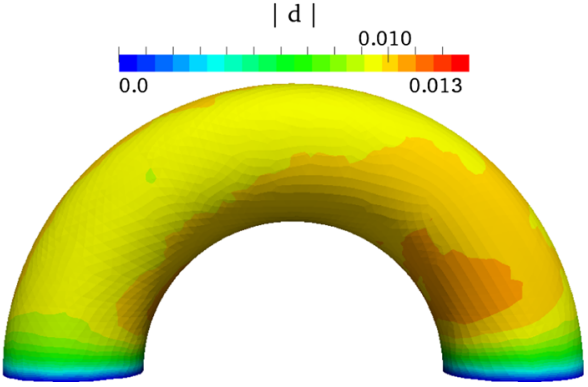

Fig. 23 Vessel wall displacement in the case of the (EXP1, EXP2-ZS) model for the study Case B. Different views. Time $t=0.100 \mathrm{~s}$

placement is oriented according to the fibers direction (clockwise or counterclockwise). Indeed, in Fig. 17a the orientation of collagen fibers indicates a counterclockwise parametrization of the main radial cut of the geometry and, as reported in Fig. 21 (right), the maximum vessel wall displacement is reached on the lateral side of the domain coherently with such parametrization. For the sake of completeness, in order to confirm that such mechanical behavior of the vessel wall is due to the employment of an anisotropic model, a sensitivity study has been carried out with respect to the direction of the collagen fibers. Specifically, two cases have been considered, represented in Fig. 17b, c. As it can be observed, the parametrization of the section in the main radial direction has been inverted with respect to Case A in Fig. 17 and rotated of $\mp 90^{\circ}$ degrees. For all three cases, the two families of fibers are characterized by the same angle, reported in Table 1, with respect to the local and main circumferential directions. The vessel wall displacement for the cases presented in Fig. 17
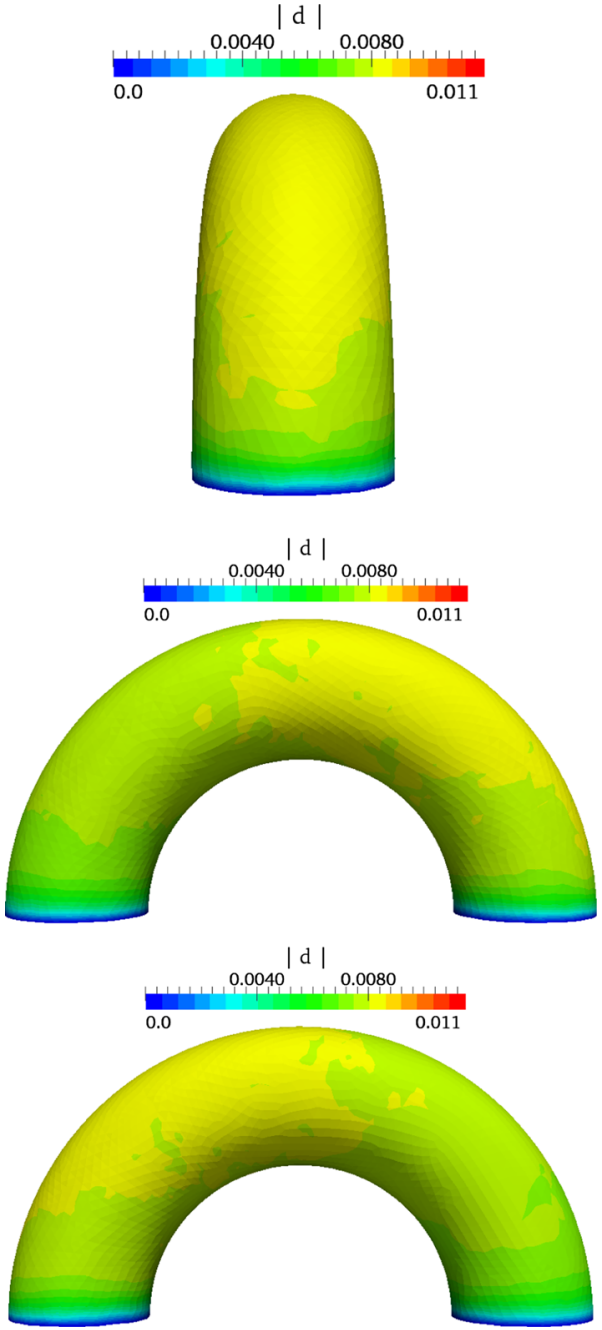

Fig. 24 Vessel wall displacement in the case of the (EXP1, EXP2-ZS) model for the study Case C. Different views. Time $t=0.100 \mathrm{~s}$

are reported in Figs. 22, 22, 23 and 24. For Cases A and B in Figs. 22 and 23, the maximum vessel wall displacement is reported on one side of the solid domain. On the other hand, in Case C in Fig. 24 (namely for a symmetric orientation of the collagen fibers with respect to the $y$ axis), the symmetry of the solid displacement $\widehat{\boldsymbol{d}}$ with respect to the $y$ axis is obtained. It is worth pointing out that, the strong dependency of the blood vessel displacement on the spatial orientation of the collagen fibers may in turn affect the spatial distribution of the mechanical stresses inside the vessel wall during the heart beat.

\section{Conclusions}

The numerical results presented in this work highlighted different aspects of the numerical simulation of the coupled FSI system. On one hand, it was found that the choice of the constitutive model for the arterial wall does not strongly affect 
the fluid dynamics inside the vessel lumen for the idealized cerebral arteries under consideration and for the relatively small values of the typical Reynolds number. On the other hand, non-negligible differences have been reported regarding the displacement fields yielded by the different constitutive models. More precisely, in the case of the straight cylinder, although the similar qualitative behavior of all the numerical solutions, it has been observed that in the physiological range of blood pressures and velocities, the isotropic first order exponential model yields the smallest vessel wall displacement, while the isotropic second order exponential law yields the highest values. Consequently, significant differences, especially through the thickness of the vessel wall, were reported for the Von Mises stress. On the contrary, the simulations of the toroidal geometry showed that the effects of the constitutive model for the vessel wall on the numerical solutions are evident both from a qualitative and quantitative point of view. More specifically, in the case of the anisotropic model the spatial orientation of the collagen fibers has a significant influence on the displacement of the arterial tissue and, therefore, on the evaluation of the mechanical stresses that are commonly related to the possible onset and development of cardiovascular diseases, as e.g. aneurysms.

Based on the numerical simulation on the cylindrical geometry with the anisotropic model with collagen recruitment at finite strains, we showed the progressive activation and deactivation of the collagen fibers throughout the vessel wall during the cardiac cycle. We highlighted that the collagen fibers situated on the internal surface of the vessel wall are mechanically active for the largest part of the heart beat, while the ones located on the external parts of the tissue reach the active state only at the peak of the systolic phase. Despite, to the best of our knowledge, an anisotropic model that captures the collagen fibers activation at finite strains has never been used in literature for FSI numerical simulations, we deem it to be a valuable option to study the role of the collagen fibers in the vessel wall dynamics. In this respect, the use of multi-layer models for the arterial tissue, i.e. models that consider the different mechanical properties of the tunica intima, media, and adventitia, would represent a further step in the numerical study of the activation and deactivation of the collagen fibers. However, this stresses the need for experimental measurements of the properties of the media and adventitia layers of the vessel wall, including a correct description of the spatial orientation, tortuosity and density of the collagen fibers in cerebral arteries. This is an area of ongoing work in our group, which we believe will represent an important step toward the use of anisotropic constitutive models with collagen recruitment at finite strain for FSI numerical simulations of vascular disease such as cerebral aneurysms. We believe such studies, which will more accurately model the spatial and temporal variation in loads on collagen fibers, will be essential for modeling the complex role of vascular cells such as fibroblasts in the mechanobiological processes of the vascular wall.

Acknowledgments We acknowledge the support of the Swiss National Supercomputing Centre (CSCS) under the project ID s 475 for providing the computational resources for the numerical simulations. P. Tricerri acknowledges the financial support of Fundação para Ciênca e a Tecnologia (FCT) of Portugal through the Research Center CEMAT-IST (under the grant SFRH/BD/51069/2010) and of the project EXCL/MATNAN/0114/2012. Prof. Quarteroni acknowledges the MATHCARD and the HP2C projects; Dr. Deparis thanks the HP2C project.

\section{References}

1. Augsburger L (2008) Fluid Mechanics of Cerebral Aneurysms and Effects of Intracranial Stents on Cerebral Aneurysmal Flow. Ph.D. thesis, École Polytechinque Fédérale de Lausanne, Lausanne

2. Baek H, Jayaraman M, Richardson P, Karniadakis G (2010) Flow instability and wall shear stress variation in intracranial aneurysms. J R Soc Interface 7:967-988

3. Balzani D (2006) Polyconvex Anisotropic Energies and Modeling of Damage Applied to Arterial Walls. Ph.D. thesis, University of Duisburg-Essen, Essen

4. Balzani D, Brinkhues S, Holzapfel G (2012) Constitutive framework for the modeling of damage in collagenous soft tissues with application to arterial walls. Comput Methods Appl Mech Eng 213-216:139-151

5. Balzani D, Neff P, Schröder J, Holzapfel G (2006) A polyconvex framewok for soft biological tissues. Adjustment to experimental data. Int J Solids Struct 43:6052-6070

6. Balzani D, Schmidt T, Schriefl T, Holzapfel G (2013) Constitutive modeling of damage mechanisms in arterial walls and related experimental studies. In: XLI APM proceedings (advanced problems in mechanics), St. Petersburg, pp 17-25

7. Bazilevs Y, Hsu M, Zhang Y, Wang W, Kvamsdal T, Hentschel S, Isaksen J (2010) Computational vascular fluid-structure interaction: methodology and application to cerebral aneurysms. Biomech Model Mechanobiol 9:481-498

8. Bazilevs Y, Hsu M, Zhang Y, Wang W, Liang X, Kvamsdal T, Brekken R, Isaksen J (2010) A fully-coupled fluid-structure interaction simulation of cerebral aneurysms. Comput Mech 46:3-16

9. Bhole A, Flynn BP, Liles M, Saeidi N, Dimarzio CA, Ruberti JW (2009) Mechanical strain enhances survivability of collagen micronetworks in the presence of collagenase: implications for load-bearing matrix growth and stability. Philoso Trans Ser A Math Phys Eng Sci 367:3339-3362

10. Brinkhues S, Klawonn A, Rheinbach O, Schröder J (2013) Augmented Lagrange methods for quasi-incompressible materialapplications to soft biological tissue. Int J Numer Methods Biomed Eng 29:332-350

11. Burton A (1954) Relation of structure to function of the tissue of the wall of blood vessels. Physiol Rev 34:619-642

12. Calvo B, Pẽna E, Martinez M, Doblaré M (2007) An uncoupled directional damage model for fibred biological soft tissues. Formulation and computational aspects. Int $\mathbf{J}$ Numer Methods Eng 69:2036-2057

13. Carew T, Vaishnav R, Patel D (1968) Compressibility of the arterial wall. Circ Res 23:61-68

14. Castro M, Putman C, Cebral J (2006) Computational fluid dynamics modeling of intracranial aneurysms: effects of parent artery segmentation on intra-aneurysmal hemodynamics. Am J Neuroradiol 27:1703-1709 
15. Cebral J, Castro M, Appanaboyina S, Putman C, Millan D, Frangi A (2005) Efficient pipeline for image-based patient-specific analysis of cerebral aneurysm hemodynamics: techniques and sensitivity. IEEE Trans Med Imaging 24:457-467

16. Cebral J, Mut F, Weir F, Putman C (2011) Association of hemodynamics characteristics and cerebral aneurysms rupture. Am J Neuroradiol 32:264-270

17. Chandra S, Raut S, Jana A, Biederman R, Doyle M, Muluk S, Finol E (2013) Fluid-structure interaction modeling of abdominal aortic aneurysms: the impact of patient-specific inflow conditions and fluid/solid coupling. J Biomech Eng 135:810011-8100114

18. Chen H, Zhu L, Hou Y, Liu Y, Kassab G (2010) Fluid-structure interaction (FSI) modeling in the cardiovascular system. In: Guccione J, Kassab G, Ratcliffe M (eds) Computational cardiovascular mechanics, modeling and applications in heart failure. Springer, New York, pp 141-157

19. Chen J, Wang S, Ding G, Yang X, Li H (2009) The effect of aneurismal-wall mechanical properties on patient-specific hemodynamic simulations: two clinical reports. Acta Mech Sin 25:677688

20. Crosetto P (2011) Fluid-Structure Interaction Problems in Hemodynamics: Parallel Solvers, Preconditioners, and Applications. Ph.D. thesis, École Polytechinque Fédérale de Lausanne, Lausanne

21. Crosetto P, Deparis S, Fourestey G, Quarteroni A (2011) Parallel algorithms for fluid-structure interaction problems in haemodynamics. SIAM J Sci Comput 33:1598-1622

22. Crosetto P, Reymond P, Deparis S, Kontaxakis D, Stergiopulos N, Quarteroni A (2011) Fluid structure interaction simulations of physiological blood flow in the aorta. Comput Fluids 43:46-57

23. Delfino A, Stergiopulos N, Moore J, Meister J (1997) Residual strain effects on the stress field in a thick wall finite element model of the human carotid bifurcation. J Biomech 30:777-786

24. Devault K, Gremaud P, Novak V, Olufsen M, Vernières G, Zhao P (2008) Blood flow in the Circle of Willis: modeling and calibration. Multiscale Model Simulat 7:888-909

25. Draney M, Herfkens R, Hughes T, Pelc N, Wedding K, Zarins C, Taylor C (2002) Quantification of vessel wall cyclic strain using cine phase contrast magnetic resonance imaging. Ann Biomed Eng 30:1033-1045

26. Ferguson G (1972) Direct measurements of mean and pulsatile blood pressure at operation in human intracranial saccular aneurysms. J Neurosurg 36:560-563

27. Fernández M, Moubachir M (2005) A Newton method using exact jacobians for solving fluid-structure coupling. Comput Struct 83:127-142

28. Flory P (1961) Thermodynamical relations for high elastic materials. Transa Farad Soc 57:829-838

29. Ford M, Nikolov N, Milner J, Lownie S, Demont E, Kalata W, Loth F, Holdsworth D, Steinman D (2008) PIV-measured versus CFD-predicted flow dynamics in anatomically realistic cerebral aneurysm models. J Biomech Eng 130:1-15

30. Formaggia L, Quarteroni A, Veneziani AE (2009) Cardiovascular mathematics, modeling and simulation of the circulatory system. MS \& A, Springer-Verlag, Berlin

31. Gambaruto A, João A (2012) Flow structures in cerebral aneurysms. Comput Fluids 65:56-65

32. Gasser T, Ogden R, Holzapfel G (2006) Hyperelastic modelling of arterial layers with distributed collagen fiber orientations. J R Soc Interface 3:15-35

33. Gasser T, Schulze-Bauer C, Holzapfel G (2002) A threedimensional finite element model for arterial clamping. J Biomech Eng 124:355-363

34. Giller C, Bowman G, Dyer H, Mootz L, Krippner W (1993) Cerebral arterial diameters during changes in blood pressure and carbon dioxide during craniotomy. Neurosurgery 32:737-741
35. Gould RA, Chin K, Santisakultarm TP, Dropkin A, Richards JM, Schaffer CB, Butcher JT (2012) Cyclic strain anisotropy regulates valvular interstitial cell phenotype and tissue remodeling in threedimensional culture. Acta Biomater 8:1710-1719

36. Gupta V, Grande-Allen KJ (2006) Effects of static and cyclic loading in regulating extracellular matrix synthesis by cardiovascular cells. Cardiovasc Res 72:375-383

37. Hartmann S, Neff P (2003) Polyconvexity of generalized polynomial-type hyperelastic strain energy functions for nearlyincompressibility. Int J Solids Struct 40:2767-2791

38. den Heijer T, Skoog I, Oudkerk M, de Leeuw FE, de Groot J, Hofman A, Bretler M (2003) Association between blood pressure levels over time and brain atrophy in the elderly. Neurobiol Aging 24:307-313

39. Hill M, Duan X, Gibson G, Watkins S, Robertson A (2012) A theoretical and non-destructive experimental approach for direct inclusion of measured collagen orientation and recruitment into mechanical models of the arterial wall. J Biomech 45:762771

40. Hoi Y, Woodward S, Kim M, Taulbee D, Meng H (2006) Validation of CFD simulations of cerebral aneurysms with implication of geometric variations. J Biomech Eng 128:844-851

41. Holzapfel G (2000) Nonlinear solid mechanics: a continuum approach for engineering. Wiley, London

42. Holzapfel G, Gasser T, Ogden R (2000) A new constitutive framework for arterial wall mechanics and a comparative study of material models. J Elast 61:1-48

43. Holzapfel G, Odgen R (2009) Constitutive modelling of passive myocardium: a structurally based framework for material characterization. Philos Trans R Soc A 367:3445-3475

44. Holzapfel G, Ogden R (2010) Constitutive modeling of arteries. Proc R Soc Lond A 466:1551-1597

45. Humphrey J (2002) Cardiovascular solid mechanics. Cells, tissues, and organs. Springer-Verlag, New York

46. Isaksen J, Bazilevs Y, Kvamsdal T, Zhang Y, Kaspersen J, Waterloo K, Romner B, Ingebrigtsen T (2008) Determination of wall tension in cerebral artery aneurysms by numerical simulation. Stroke 39:3172-3178

47. Janela J, Moura A, Sequeira A (2010) Absorbing boundary conditions for a 3D non-Newtonian fluidstructure interaction model for blood flow in arteries. Int J Eng Sci 48:1332-1349

48. Jeong W, Rhee K (2012) Hemodynamics of cerebral aneurysms: computational analyses of aneurysm progress and treatment. Comput Math Methods Med 2012:1-11

49. Kim C, Kiris C, Kwak D, David T (2006) Numerical simulation of local blood flow in the carotid and cerebral arteries under altered gravity. J Biomech Eng 128:194-202

50. Li D, Robertson A (2009) A structural multi-mechanism constitutive equation for cerebral arterial tissue. Int J Solids Struct 46:2920 2928

51. Li D, Robertson A (2009) A structural multi-mechanism damage model for cerebral arterial tissue. J Biomech Eng 131:101013101018

52. Li D, Robertson A, Lin G, Lovell M (2012) Finite element modeling of cerebral angioplasty using a structural multi-mechanism anisotropic damage model. Int J Numer Methods Eng 92:457-474

53. Lou J, Lee D, Morsi H, Mawad M (2008) Wall shear stress on ruptured and unruptured intracranial aneurysms at the internal carotid artery. Am J Neuroradiol 29:1761-1767

54. Malossi A (2012) Partitioned Solution of Geometrical Multiscale Problems for the Cardiovascular System: Models, Algorithms, and Applications. Ph.D. thesis, École Polytechinque Fédérale de Lausanne, Lausanne

55. Malossi A, Bonnemain J (2013) Numerical comparison and calibration of geometrical multiscale models for the simulation of arterial flows. Cardiovasc Eng Tecnol 4:440-463 
56. Mantha A, Karmonik C, Bendorf G, Strother C, Metcalfe R (2006) Hemodynamics in a cerebral artery before and after the formation of an aneurysm. Am J Neuroradiol 27:1113-1118

57. Marks M, Pelc N, Ross M, Enzmann D (1992) Determination of cerebral blood flow with a phase contrast cine MR imaging technique: evaluation of normal subjects and patients with an arteriovenous malformations. Radiology 182:467-476

58. Marzo A, Singh P, Reymond P, Stergiopulos N, Patel U, Hose R (2009) Influence of inlet boundary conditions on the local haemodynamics of intracranial aneurysms. Comput Methods Biomech Biomed Eng 12:431-444

59. Moireau P, Xiao N, Astorino M, Figueroa C, Chapelle D, Taylor C, Gerbeau J (2012) External tissue support and fluid-structure simulation in blood flows. Biomech Model Mechanobiol 11:1-18

60. Nichols W, O'Rourke M (1998) McDonald's blood flow in arteries: theoretical, experimental, and clinical principles. Arnold, London

61. Nobile F (2001) Numerical Approximation of Fluid-Structure Interaction Problems with Application to Haemodynamics. Ph.D. thesis, École Polytechinque Fédérale de Lausanne, Lausanne

62. Oshima M, Sakai H, Torii R (2005) Modelling of inflow boundary conditions for image-based simulation of cerebrovascular flow. Int J Numer Methods Fluids 47:603-617

63. Quarteroni A, Sacco R, Saleri F (2007) Numerical mathematics. Springer, Berlin

64. Quarteroni A, Valli A (1999) Domain decomposition methods for partial differential equations. Oxford University Press, Oxford

65. Quarteroni A, Valli A (1999) Numerical approximation of partial differential equations. Springer-Verlag, Berlin

66. Reymond P, Bohraus Y, Perren F, Lazeyras F, Stergiopulos N (2010) Validation of a patient-specific one-dimensional model of the systemic arterial tree. Am J Physiol 301:1173-1182

67. Roach M, Burton A (1957) The reason for the shape of the distensibility curves of arteries. Can J Biochem 35:681-690

68. Robertson A, Sequeira A, Kameneva M (2008) Hemorheology. In: Galdi G, Rannacher R, Robertson A, Turek S (eds) Hemodynamical flows, oberwolfach seminars, vol 37. Springer-Verlag, Basel, pp $63-120$

69. Robertson A, Watton P (2013) Mechanobiology of the arterial wall. In: Becker S, Kuznetsov A (eds) Modeling of transport in biological media. Elsevier, New York, pp 275-347

70. Roy S, Boss C, Rezakhaniha R, Stergiopulos N (2010) Experimental characterization of the distribution of collagen fiber recruitment. J Biorheol 24:84-93

71. Saad Y (2003) Iterative methods for sparse linear systems. SIAM, Philadelphia

72. Schröder J, Neff P (2003) Invariant formulation of hyperelastic transverse isotropy based on polyconvex free energy functions. Int J Solids Struct 40:401-445

73. Scott S, Ferguson G, Roach M (1972) Comparison of the elastic properties of human intracranial arteries and aneurysms. Can J Physiol Pharmacol 50:328-332

74. Sforza D, Putman C, Cebral J (2009) Hemodynamics of cerebral aneurysms. Annu Rev Fluid Mech 41:91-107

75. Spencer A (1984) Constitutive theory of strongly anisotropic solids. In: Spencer A (ed) Continuum theory of the mechanics of fibrereinforced composites. Springer-Verlag, Wien, pp 1-32
76. Takizawa K, Bazilevs Y, Tezduyar T, Long C, Marsden A, Schjodt K (2014) Patient-specific cardiovascular fluid mechanics analysis with the ST and ALE-VMS methods. In: Idelsohn S (ed) Numer Simulat Coupled Probl Eng, vol 33. Springer, Heidelberg, pp 71102

77. Takizawa K, Bazilevs Y, Tezduyar T (2012) Space-time and ALEVMS techniques for patient-specific cardiovascular fluid-structure interaction modeling. Arch Comput Methods Eng 19:171-225

78. Takizawa K, Takagi H, Tezduyar T, Torii R (2014) Estimation of element-based zero-stress state for arterial FSI computations. Comput Mech 54:895-910

79. Tezduyar T, Takizawa K, Brummer T, Chen P (2013) Spacetime fluidstructure interaction modeling of patient-specific cerebral aneurysms. Numer Methods Biomed Eng 27:1665-1710

80. Torii R, Oshima M, Kobayashi T, Takagi K, Tezduyar T (2006) Fluid-structure interaction modeling of anuerysmal conditions with high and normal blood pressures. Comput Mech 38:482-490

81. Torii R, Oshima M, Kobayashi T, Takagi K, Tezduyar T (2007) Numerical investigation of the effect of hypertensive blood pressure on cerebral aneurysm-dependence of the effect on the aneurysm shape. Int J Numer Methods Fluids 54:995-1009

82. Torii R, Oshima M, Kobayashi T, Takagi K, Tezduyar T (2008) Fluidstructure interaction modeling of a patient-specific cerebral aneurysm: influence of structural modeling. Comput Mech 43:151159

83. Torii R, Oshima M, Kobayashi T, Takagi K, Tezduyar T (2009) Fluid-structure interaction modeling of blood flow and cerebral aneurysm: significance of artery and aneurysm shapes. Comput Method Appl Mech Eng 198:3613-3621

84. Torii R, Oshima M, Kobayashi T, Takagi K, Tezduyar T (2010) Influence of wall thickness on fluid-structure interaction computations of cerebral aneurysms. Int J Numer Methods Biomed Eng 26:336-347

85. Tricerri P, Dedè L, Quarteroni A, Sequeira A (2013) Numerical validation of isotropic and transversely isotropic constitutive models for healthy and unhealthy cerebral arterial tissue. Technical Report 39.2013, MATHICSE Report EPFL

86. Valencia A, Burdiles P, Ignat M, Mura J, Bravo E, Rivera R, Sordo J (2013) Fluid structural analysis of human cerebral aneurysm using their own wall mechanical properties. Comput Math Methods Med http://www.hindawi.com/journalscmmm/2013/293128/

87. Valencia A, Solis F (2006) Blood flow dynamics and arterial wall interaction in a saccular aneurysm model of the basilar artery. Comput Struct 84:1326-1337

88. Weisbecker H, Pierce D, Holzapfel G (2011) Modeling of damageinduced softening for arterial tissue. In: Proceedings of the 2011 SCATh joint workshop on new tecnologies for computer/robot assisted surgery, Graz, pp 1-4

89. Wulandana R, Robertson A (2005) An inelastic multi-mechanism constitutive equation for cerebral arterial tissue. Biomech Model Mechanobiol 4:235-248

90. Zakaria H, Robertson A, Kerber C (2008) A parametric model for studies of flow in arterial bifurcations. Ann Biomed Eng 36:15151530 